\title{
A novel oral micellar fenretinide formulation with enhanced bioavailability and antitumour activity against multiple tumours from cancer stem cells
}

Isabella Orienti ${ }^{1+}$, Valentina Salvati ${ }^{2,3+}$, Giovanni Sette ${ }^{2,3+}$, Massimo Zucchetti ${ }^{4}$, Lucilla Bongiorno-Borbone ${ }^{5}$, Angelo Peschiaroli ${ }^{6}$, Lello Zolla ${ }^{7}$, Federica Francescangeli ${ }^{2}$, Mariella Ferrari ${ }^{4}$, Cristina Matteo ${ }^{4}$, Ezia Bello ${ }^{4}$, Antonio Di Virgilio ${ }^{8}$, Mario Falchi ${ }^{9}$, Maria Laura De Angelis ${ }^{2}$, Marta Baiocchi ${ }^{2}$, Gerry Melino ${ }^{5}$, Ruggero De Maria ${ }^{3,10}$, Ann Zeuner ${ }^{2+}$ and Adriana Eramo ${ }^{2 *}$ (B)

\begin{abstract}
Background: An increasing number of anticancer agents has been proposed in recent years with the attempt to overcome treatment-resistant cancer cells and particularly cancer stem cells (CSC), the major culprits for tumour resistance and recurrence. However, a huge obstacle to treatment success is the ineffective delivery of drugs within the tumour environment due to limited solubility, short circulation time or inconsistent stability of compounds that, together with concomitant dose-limiting systemic toxicity, contribute to hamper the achievement of therapeutic drug concentrations. The synthetic retinoid Fenretinide (4-hydroxy (phenyl)retinamide; 4-HPR) formerly emerged as a promising anticancer agent based on pre-clinical and clinical studies. However, a major limitation of fenretinide is traditionally represented by its poor aqueous solubility/bioavailability due to its hydrophobic nature, that undermined the clinical success of previous clinical trials.

Methods: Here, we developed a novel nano-micellar fenretinide formulation called bionanofenretinide (Bio-nFeR), based on drug encapsulation in an ion-pair stabilized lipid matrix, with the aim to raise fenretinide bioavailability and antitumour efficacy.
\end{abstract}

Results: Bio-nFeR displayed marked antitumour activity against lung, colon and melanoma CSC both in vitro and in tumour xenografts, in absence of mice toxicity. Bio-nFeR is suitable for oral administration, reaching therapeutic concentrations within tumours and an unprecedented therapeutic activity in vivo as single agent.

Conclusion: Altogether, our results indicate Bio-nFeR as a novel anticancer agent with low toxicity and high activity against tumourigenic cells, potentially useful for the treatment of solid tumours of multiple origin.

Keywords: Fenretinide, Bioavailability, Solubility, Antitumour activity, Cancer stem cells, Solid tumours, Drug delivery, Pharmacokinetics, Cancer therapy

\footnotetext{
* Correspondence: adriana.eramo@iss.it

†'sabella Orienti, Valentina Salvati, Giovanni Sette, Ann Zeuner and Adriana

Eramo contributed equally to this work.

${ }^{2}$ Department of Oncology and Molecular Medicine, Istituto Superiore di

Sanità, Rome, Italy

Full list of author information is available at the end of the article
}

(c) The Author(s). 2019 Open Access This article is distributed under the terms of the Creative Commons Attribution 4.0 International License (http://creativecommons.org/licenses/by/4.0/), which permits unrestricted use, distribution, and reproduction in any medium, provided you give appropriate credit to the original author(s) and the source, provide a link to the Creative Commons license, and indicate if changes were made. The Creative Commons Public Domain Dedication waiver (http://creativecommons.org/publicdomain/zero/1.0/) applies to the data made available in this article, unless otherwise stated. 


\section{Background}

Most tumours are heterogeneous and contain a small population of CSC that are believed to play a crucial role in tumour progression, drug resistance, recurrence and metastasis in multiple malignancies [30]. Thus, several potential approaches to kill therapeutically-resistant CSC have been deeply explored and, besides overcoming primary intrinsic resistance of tumour cells, they have been proved effective against tumour relapse and metastasis, as well [12, 22]. However, in view of the plasticity of tumour cells, drugs with broad toxicity against different tumour cell populations would to be preferred to achieve long-term antitumour efficacy [40]. Another huge obstacle to treatment efficacy is the inefficient drug absorption into the tumour environment mainly due to low systemic availability which limits the drug distribution to the different body compartments, including the tumor. Moreover, the unspecific toxicity of the antitumor drugs strongly restrains dose-escalation as a mean to improve the systemic availability [45]. Properly designed nanoformulations may offer the possibility of increasing the drug systemic availability, thus improving the drug concentration at the tumour site to levels suitable at eliciting an antitumour response.

In nanoformulations the drug is generally enclosed in nanostructured materials (nanoparticles) providing drug aqueous solubilization at extents depending on the overall characteristic of the system. Based on the physico-chemical properties of the materials the nanoparticles may be nanomicelles, nanocapsules, nanospheres etc. [23]. They are mainly administered by oral or intravenous route. In intravenous administration the nanoparticle role changes with the tumor characteristics. In tumors with vascular discontinuities the nanoparticle function is to transport the drug in circulation avoiding release until extravasation takes place through the discontinuities of the tumor capillaries where the nanoparticle accumulation provides increased drug concentration in the tumor environment with respect to the other body compartments [18, 25, 34].

In oral administration the nanoparticle role is to increase the drug solubilization and absorption in the gastrointestinal environment to enhance drug plasma concentration and biodistribution at suitable levels for drug activity. The nanoparticles do not cross the gastrointestinal wall and lipophilic drugs such as fenretinide are primarily absorbed by the lipid absorption pathway mediated by chylomicrons (Barry J. [26]).

Nanoparticles for oral administratioin have been extensively studied to improve the therapeutic efficacy of antitumor drugs [11]. Oral lipid nanoparticles, in particular have been proved to increase solubility, chemical stability, epithelium permeability and bioavailability of hydrophobic bioactive compounds [17].
Moreover, lipid nanocarriers offer new possibilities of obtaining therapeutic drug levels by oral administration, the most simple and reliable administration route. Indeed they have been proved to increase solubility, chemical stability, epithelium permeability and bioavailability of hydrophobic bioactive compounds [17].

Fenretinide (4-HPR) is a synthetic retinoid which emerged as a promising anticancer agent based on numerous in vitro and animal studies as well as chemoprevention clinical trials (B. J. $[29,48,49])$. It is reported to induce cytotoxicity by multiple mechanisms (Holliday, Cox, Kang, \& [27]; B. J. [28, $35,38,51,52])$. Significantly, fenretinide has shown the potential to target CSC-associated molecular targets, further increasing its therapeutic value $[1,10,33,53]$. An oral formulation of fenretinide, consisting of soft gelatin capsules containing fenretinide in corn oil and polysorbate, is currently available at the National Cancer Institute (NCI-FeR). However, despite promising in vitro cytotoxicity, its antitumour activity in clinical trials (both as a cancer therapeutic and chemopreventive agent) remains limited, likely due to poor bioavailability of this drug formulation requiring the administration of excessively elevated drug doses that constrain drug tolerability and patient compliance $[3,4,15]$. Numerous attempts have been made to develop fenretinide formulations capable of increasing the drug aqueous solubility and bioavailability to levels that can elicit a satisfactory therapeutic response. Promising, although still unsatisfactory results have been obtained in a trial with neuroblastoma children, while in adult patients (lymphoma) the high drug doses required to achieve therapeutic plasma drug concentrations strongly limited patient compliance causing gastrointestinal toxicity and (reversible) nyctalopia. Improved efficacy of oral fenretinide, when co-administered with other drugs, has been reported, possibly due to lower drug concentration required for synergistic activity or to increased fenretinide exposure induced by co-administered drugs [4, $8,20,27,37]$. Intravenously infused fenretinide as a soy oilin-water emulsion showed promising results in patients with hematologic malignancies [32]. Lastly, all these studies suggested that improved fenretinide formulations are needed to couple acceptable drug doses, tolerability and patient compliance with significant systemic exposure, aiming at satisfactory therapeutic efficacy also as single agent treatment for both adult and pediatric patients.

We recently reported a new fenretinide-cyclodextrin complex, which has a higher solubility and bioavailability as compared to previous formulations [36]. However such complex requires intravenous administration, which limit the potential clinical use of this drug particularly for extended treatment periods. Now, we developed a nano-formulation of fenretinide, called bionanofenretinide (Bio-nFeR) with the aim to combine a high bioavailability and low toxicity with the suitability for oral administration. 
We developed a new nano-formulation of fenretinide, called bionanofenretinide (Bio-nFeR) designed with the aim to raise the poor oral fenretinide bioavailability, that has prevented its clinical use in spite of its considerable antitumour activity and low toxicity profile, until now. Bio-nFeR is based on ion pair formation between fenretinide and phosphatidylcholine triggering the spontaneous formation of micelles, in water, containing fenretinide in their inner core.

Bio-nFeR displayed very promising antitumour activity, superior than NCI-FeR, against CSC of various origin in vitro at low concentrations, and in CSC-derived tumour xenografts of lung, colon and melanoma origin, reaching higher drug concentration in blood and therapeutic drug tumour levels within xenografts, in absence of toxicity.

\section{Materials and methods}

\section{Chemicals}

4-HPR (fenretinide) was purchased from Olon Spa (Milan Italy). L- $\alpha$-phosphatidylcholine from egg yolk, glyceryl tributyrate and all the other chemicals were purchased from Sigma-Aldrich. Deuterated fenretinide $\left(\mathrm{C}_{26} \mathrm{H}_{29} \mathrm{D}_{4} \mathrm{NO}_{2}\right)$ used as internal standard (IS), was obtained from Tocris Bioscience (Bristol, UK).

\section{Bio-nFeR preparation}

Fenretinide was homogeneously mixed with L- $\alpha$-phosphatidylcholine and glyceryl tributyrate dispersed in alkaline ethanol to a final weight ratio 1:9:1 w:w:w respectively. Ethanol was removed from the mixture in a rotary evaporator and the dry residue stored at $-18^{\circ} \mathrm{C}$ until use. Reconstitution of the dry residue to Bio-nFeR nanomicelles was accomplished by dissolving the residue in water at $30^{\circ} \mathrm{C}$ in an ultrasound bath where a wave frequency of $40 \mathrm{kHz}$ was applied for $1 \mathrm{~h}$ to the dissolving phase. The dissolved residue was subsequently filtered through 0.4 um pore filters to obtain homogeneously dispersed nanomicelles of controlled mean size. To compare Bio-nFeR with the soft gelatin capsules representing the standard formulation most used in clinical trials for fenretinide, we reconstituted the capsules content according to the directions provided by National Cancer Institute per capsule (fenretinide: $100 \mathrm{mg}$, corn oil: 704 $\mathrm{mg}$, polysorbate $80: 60 \mathrm{mg}$ ). The final concentration in the reconstituted content of the NCI capsules (NCI-FeR) being $11.6 \% \mathrm{w}: \mathrm{w}$ fenretinide:internal capsule components, as per the NCI instructions.

\section{Solubilization ability, encapsulation efficiency and drug loading capacity of bio-nFeR towards fenretinide}

To evaluate the solubilization ability of Bio-nFeR towards fenretinide, reconstitution of the dry residue to nanomicelles was carried out at concentrations increasing from 50 to $300 \mathrm{mg} / \mathrm{ml}$, corresponding to the concentration range used in this study. After filtration through 0.4 um pore filters, the nanomicelles were diluted (1:3, v:v) with an ethanol:water (1:1, v:v) mixture and spectrophotometrically analysed at $364 \mathrm{~nm}$ for their fenretinide content. As a comparison the same evaluation was carried out on empty nanomicelles prepared by the same procedure. The encapsulation efficiency was obtained as the ratio between the drug content in the nanomicelles and the drug loaded in the dry residue used for the nanomicelles preparation. The drug loading capacity was calculated as the drug weight content in the nanomicelles per unit weight of nanomicelles, obtained by the weight of the freeze-dried nanomicelle suspensions.

\section{Light scattering characterization of bio-nFeR}

Particle size, polydispersity and zeta potential were measured at $37^{\circ} \mathrm{C}$ on nanomicelle dispersions obtained by reconstitution of the dry residue at $50 \mathrm{mg} / \mathrm{ml}$ and diluted 1:100 with water (Malvern Instruments, Worcestershire, UK). A minimum of 12 measurements per sample were made. Results were the combination of three 10-min runs for a total accumulation correlation function time of $30 \mathrm{~min}$. The results were volume-weighted.

\section{Feneretinide release from bio-nFeR}

The release of fenretinide from Bio-nFeR was measured in three different media: $\mathrm{HCl}$ solution ( $\mathrm{pH} 1.2)$, phosphate buffer solution ( $\mathrm{pH} 6.8$ ) and phosphate buffer solution ( $\mathrm{pH}$ 6.8) containing sodium taurocholate (3.0 $\mathrm{mM}$ ), to simulate the main physico-chemical characteristics of the gastrointestinal fluids [19]. The nanomicelles obtained by reconstitution of the dry residue at $50 \mathrm{mg} /$ $\mathrm{ml}$ were diluted (1:10) with the different media and placed $(1 \mathrm{ml})$ in a dialysis bag $(\mathrm{Mw}$ cutoff $5 \mathrm{KD})$ which was subsequently sealed and immersed in a receiving compartment containing water $(20 \mathrm{ml})$ and n-octanol (5 $\mathrm{ml}$ ). N-octanol was added as a drug-extraction phase simulating the presence of cell membranes in vivo [31]. The system was thermostated at $37^{\circ} \mathrm{C}$. At increasing time intervals spectrophotometric analysis in the releasing compartment was carried out as described above.

\section{Confocal microscopy}

Images were taken by a FV1000 confocal microscope (Olympus, Tokyo, Japan), using a (Olympus) plan apo objective $60 \times$ oil A.N. 1,42. Excitation light for fenretinide was obtained by an Argon Ion Laser ( $488 \mathrm{~nm}$ ) and emission was recorded from 495 to $550 \mathrm{~nm}$. Images were taken also by a Nikon $\mathrm{C} 1 \mathrm{~s}$ confocal laser scanning microscope, equipped with a Nikon PlanApo 60, 1.4-NA oil immersion lens. 
Cell culture, drug treatments and cell viability assay Patient-derived CSC lines were obtained from primary, melanoma, glioblastoma, lung and colon cancers and cultured as previously described [9, 14, 42-44].

For drug treatments and cell viability assays, 3000 cells were plated in triplicate in 96-well plates and left untreated or exposed for $72 \mathrm{~h}$ to Bio-nFeR (stock solution $200 \mathrm{mg} / \mathrm{ml}$ freshly dissolved in water) or NCI-FeR (20 $\mathrm{mg} / \mathrm{ml}$ dissolved in corn oil and polisorbate 80 ). Cell viability was detected with Cell TiterGlo (Promega).

\section{Pharmacokinetic studies Animals}

The experiments were performed in CD1 female mice, 7 weeks of age, (body weight $25 \pm 2 \mathrm{~g}$ ) obtained from Envigo RMS SrL (Udine, Italy). They were maintained under specific-pathogen-free conditions with constant temperature and humidity, according to institutional guidelines regularly checked by a certified veterinarian. Animal experimentation was conducted in conformance with the following laws, regulations, and policies governing the care and use of laboratory animals: Italian Governing Law (D. 1. 26/2014; Authorization n.19/2008-A issued March 6, 2008 by the Ministry of Health). Mario Negri Institutional Regulations and Policies providing internal authorizations for persons conducting animal experiments (Quality Management System Certificate -UNI EN ISO 9001:2008 - Reg. N 86121). The experimental protocol was reviewed by the Mario Negri Institute Ethical Committee and approved by the Italian Ministry of Health (Aut. Min. No 489/2016-Pr).

\section{Drug and formulations}

The pharmacokinetics of oral Bio-nFeR was investigated in comparison to that of the reference formulation NCIFeR, after single administration of multiple doses and after chronic administrations, daily for 2 weeks or bidaily for 1 week. Bio-nFeR was reconstituted at $20 \mathrm{mg} / \mathrm{mL}$ fenretinide to allow the administration of $200 \mathrm{mg} / \mathrm{Kg}$ fenretinide that corresponded to the highest dose. The doses of 100,50 and $10 \mathrm{mg} / \mathrm{Kg}$ were administered by using serial dilution of the $20 \mathrm{mg} / \mathrm{mL}$ solution.

NCI-FeR prepared, as per the NCI instructions, at $11.6 \% \mathrm{w}: \mathrm{w}$ fenretinide:internal capsule components, was diluted with corn oil to obtain the treatment solutions at the concentrations of 20,15 and $10 \mathrm{mg} / \mathrm{mL}$, corresponding to the administered doses of 200, 150 and $100 \mathrm{mg} / \mathrm{Kg}$ respectively.

\section{Treatments and samples collection. Acute study}

The oral pharmacokinetics of Bio-nFeR were investigated in four independent groups of 24 mice, randomized to receive different treatment with the following fenretinide equivalent doses: 10, 50, 100 and $200 \mathrm{mg} / \mathrm{Kg}$.
The drug was administered by oral gavage and after treatment, blood samples were taken at 15 and $30 \mathrm{~min}$ and at 1,2, 4, 8/10, 24 and $48 \mathrm{~h}$ and collected in heparinized tubes from the retro-orbital plexus of the mice under isoflurane anesthesia. Mice were then sacrificed by cervical dislocation and liver, kidney and brain were removed and immediately frozen in dry-ice for future determination. To obtain plasma, blood was centrifuged at $4000 \mathrm{rpm}$ for $10 \mathrm{~min}$ at $4{ }^{\circ} \mathrm{C}$. To evaluate fenretinide and metabolites excretion, urine and faeces were collected over a $0-24 \mathrm{~h}$ and $24-48 \mathrm{~h}$ periods. The collection was performed in three mice per group of treatment, individually housed into metabolic cages for urine and faeces collection. All the biological samples were stored at $-20^{\circ} \mathrm{C}$ until analysis. To compare the absorption of Bio-nFeR to NCI-FeR and to study the bioavailability, another independent groups of 24 mice were randomized to receive an oral dose of $200 \mathrm{mg} / \mathrm{Kg}$ of NCI-FeR. Blood samples were taken at the time reported above. Twenty-four hours fractions of urine and faeces were collected in three mice per group of treatment. Plasma and the other biological specimens were stored at $-20^{\circ} \mathrm{C}$ until analysis. Chronic study: the oral pharmacokinetic of Bio$\mathrm{nFeR}$ in comparison with NCI-FeR were also investigated after chronic administrations. Mice were divided in three independent groups, randomized to receive a single treatment of $150 \mathrm{mg} / \mathrm{Kg}$ or different repeated treatments of $150 \mathrm{mg} / \mathrm{Kg}$, daily for 2 weeks (12 days on and 2 days off), or $100 \mathrm{mg} / \mathrm{Kg}$ bi-daily for 1 week ( 5 days on 2 days off). Bio-nFeR and NCI-FeR were administered by oral gavage, collecting blood samples at $0.5,1,2,4,8 / 10$ and $24 \mathrm{~h}$, the day of the single treatment and at $0,1,2,4,8 / 10,24$ and $48 \mathrm{~h}$ after the last dose of both the chronic treatments. The procedures of blood collection, plasma preparation, sacrifice of mice and storing of samples were as reported in acute study. Also during chronic treatment, excretions of fenretinide and metabolites in urine and faeces were determined collecting the specimens in three mice per group, both at the start of treatment and at the end of the repeated administration. All the collected samples were stored at $-20^{\circ} \mathrm{C}$ until analysis.

\section{Drug assay and pharmacokinetic elaboration}

The determination of fenretinide in plasma of mice and the others biological samples was determined according to a method specifically developed and validated for the quantification of fenretinide (4-HPR) based on liquid chromatography-tandem mass spectrometry (LC-MS/MS) (manuscript in preparation). The procedure of sample preparation and quantification can be summarized as follows. $30 \mu \mathrm{L}$ aliquot of plasma was added with $3 \mathrm{ng}$ of deuterated internal standard $\left({ }^{2} \mathrm{H}_{4} 4-\mathrm{HPR}\right)$, deproteinized by mean of $90 \mu \mathrm{L}$ of acetonitrile and centrifuged $5 \mathrm{~min}$ at $13200 \mathrm{rpm}$ at room temperature. The supernatant was 
recovered and $5 \mu \mathrm{L}$ injected into a HPLC system (Series 200, Perkin Elmer, MA, USA). Chromatographic separation was achieved on a Gemini-C18 column $(50 \mathrm{~mm} \times 2.0 \mathrm{~mm}, 5 \mu \mathrm{m}$ particle size; Phenomenex Inc., Torrance, CA, USA) at $32^{\circ} \mathrm{C}$ protected with a Security Guard ${ }^{\mathrm{mm}}$ ULTRA cartridges C18. The detection obtained by Mass spectrometric detection was carried out on a triple quadrupole API 4000 mass spectrometer (Sciex, MA, USA) equipped with atmospheric pressure chemical ionization source (APCI) operating in positive ion mode monitoring the transitions $392.3>283.2 \mathrm{~m} / \mathrm{z}$ for 4 HPR and $396.3>283.2 \mathrm{~m} / \mathrm{z}$ for the deuterated internal standard. To assay study samples, a calibration curve of six points (range: $1.0-500.00 \mathrm{ng} / \mathrm{mL}$ ) was prepared in control mouse plasma assessing the precision and the accuracy of the run analyzing at the same time a set of freshly prepared quality controls samples. The Limit Of Quantification of the method was $1 \mathrm{ng} / \mathrm{mL}$ in plasma and urine and $50 \mathrm{ng} / \mathrm{g}$ in faeces.

\section{Xenografts generation, antitumour activity studies and tumour pharmacokinetics of bio-nFeR}

Cell suspensions of lung and colon cancer and melanoma CSC lines were mixed 1:1 with Growth Factor-Reduced Matrigel (Beckton Dickinsons) and injected subcutaneously in both flanks of 5-week-old female NOD-SCID (non-obese diabetic/severe combined immunodeficiency) mice in the case of colon and melanoma or NSG (non-obese diabetic/ severe combined immunodeficiency gamma chain deficient) mice in the case of lung () (Charles River, Wilmington, MA, USA). For drug treatment, when tumours reached a mean diameter of $0.5 \mathrm{~cm}$, groups of 6 mice were randomized into 3 groups and treated with vehicle, with Bio-nFeR $(50,100$ or $150 \mathrm{mg} / \mathrm{kg}$ as described) freshly dissolved in sterile water or with the standard formulation NCI-FeR that reproduce NCI capsule (50 or $100 \mathrm{mg} / \mathrm{Kg}$ ). Treatments were administered by oral gavage 5 days on and 2 days off for 3 weeks. Tumours were measured twice a week using a caliper, and mice were monitored for signs of drug-induced toxicity and weighed regularly. At the end of treatments, in a study paralleling the assessment of antitumour activity, tumours and blood samples were collected for pharmacokinetic and molecular studies 3 hours after the last drug administration. Collection of blood, plasma separation and storing were as previously described. Immediately after blood collection mice were sacrificed by cervical dislocation, tumours removed rapidly frozen in liquid nitrogen and stored in small pieces at $-80^{\circ} \mathrm{C}$ for subsequent fenretinide determination, protein and lipid analyses. All procedures were performed with the approval of the Ethics Committee for Animal Experimentation.

\section{Lipid analyses: HPLC-HRMS}

Extracted tissue samples $(20 \mu \mathrm{l})$ were injected into an Ultra High Performance Liquid Chromatography (UHPLC) system (Ultimate 3000, Thermo). Samples were loaded onto a
Reprosil C18 column $(2.0 \mathrm{~mm} \times 150 \mathrm{~mm}, 2.5 \mu \mathrm{m}-\mathrm{Dr}$. Maisch, Germany) for lipids separation. Chromatographic separations were achieved at a column temperature of $30^{\circ} \mathrm{C}$; and flow rate of $0.2 \mathrm{~mL} / \mathrm{min}$. For lipids multi-step gradient program was used. It started with $8 \%$ solvent A (ddH20, 20 mmol L-1 ammonium formiate; $\mathrm{pH} 5$ ) to $6 \%$ solvent A for $3 \mathrm{~min}$ than to $2 \%$ solvent $\mathrm{A}$ for $35 \mathrm{~min}$ and finally to $100 \%$ solvent B (methanol) in $30 \mathrm{~min}$. At the end of gradient, the column was reconditioned with $8 \%$ solvent A for $5 \mathrm{~min}$.

The UHPLC system was coupled online with a mass spectrometer Q- Exactive (Thermo). The instrument was used with full scan (FS) and a subsequent data dependent acquisition (DDA) mode. The settings for Full scan were as follows: resolution, 70,000; automatic gain control (AGC) target, 3e6; maximum injection time (IT), $100 \mathrm{~ms}$; and scan range, $\mathrm{m} / \mathrm{z} 150-2000$. The remaining settings for DDA mode were as follows: resolution, 17,500; AGC target, 1e5; maximum IT, $50 \mathrm{~ms}$; isolation window, m/z1.0, HCD with stepped normalized collision energy (NCE), 30. Calibration was performed before each analysis against positive or negative ion mode calibration mixes (Piercenet, Thermo Fisher, Rockford, IL) to ensure sub ppm error of the intact mass. Lipid identification was performed with LipidSearch software (Thermo) that processes LC-MS data, to make provide accurate lipid identification.

\section{Immunofluorescence and confocal analysis of xenografts}

Tumour tissue samples were collected in oct, frozen on dry ice and stored at $-80^{\circ} \mathrm{C}$ until further use. Six micron tissue sections were cut with cryostat and mounted on coverslips. Tissue sections were fixed with $2 \%$ paraformaldehyde, permeabilized with $0.1 \%$ Triton X-100/ PBS, quenched with $1 \mathrm{M}$ glycine in PBS and incubated with antibodies to KI-67 (Dako, clone MIB-1) followed by secondary anti-mouse antibody coupled with Alexa 488 (Invitrogen). TUNEL assay was performed using in situ cell death detection KIT fluorescein (Roche) following the manufacturer's instructions. Nuclei were counterstained with DAPI (Invitrogen) and slides were permanently mounted. Images were taken by a FV1000 confocal microscope (Olympus, Tokyo, Japan), using a (Olympus) objective 10x. Excitation light was obtained by a Laser Dapi $408 \mathrm{~nm}$ for DAPI, an Argon Ion Laser $(488 \mathrm{~nm})$ for Alexa 488. DAPI emission was recorded from 415 to $485 \mathrm{~nm}$, FITC emission was recorded from 495 to $550 \mathrm{~nm}$.

\section{Flow cytometry and aldefluor assay}

For flow cytometry, $1 \times 10^{5}$ cells were incubated with the APC-conjugated monoclonal mouse IgG1 antibody anti CD44V6 Clone \# 2F10 R\&D System or negative control APC-conjugated mouse IgG1 (Ebioscience). Alternatively, 
the equivalent PE-conjugated antibodies were used. Stained cells were analyzed with a FACScanto flow cytometer (Becton Dickinson). The stem cell population expressing ALDH enzymatic activity was detected by means of the Aldefluor ${ }^{\mathrm{Tx}} k i t$ (StemCell Technologies, Vancouver, $\mathrm{BC}$, Canada), according to the manufacturer's instructions. Briefly, $1 \times 10^{5}$ cells were incubated in Aldefluor assay buffer containing ALDH-substrate for $45 \mathrm{~min}$ at $37^{\circ} \mathrm{C}$; negative control cells were stained using identical conditions in the presence of ALDH inhibitor diethylaminobenzaldehyde (DEAB). Samples were analyzed by FACScanto flow cytometer (Becton Dickinson) and the fluorescence profiles were compared.

\section{Immunoblot analyses}

For immunoblotting studies, $20 \mu \mathrm{g}$ of proteins from each sample were resolved on 4-12\% polyacrylamide gel electrophoresis NuPAGE Bis-Tris (Invitrogen, Carlsbad, CA, USA) and transferred to nitrocellulose membranes. The following primary antibodies were used: CD44v6 (VFF18) mouse monoclonal (eBioscience $\left.{ }^{\mathrm{Tm}}\right)$, c-Kit (D13A2) rabbit polyclonal (Cell Signaling), ALDH1 (clone44/ ALDH) mouse monoclonal (Beckton Dikinsons), BMI1 (D20B7) rabbit polyclonal (Cell Signaling), SOX2 (D6D9) rabbit polyclonal (Cell Signaling) and $\beta$-actin monoclonal (Sigma-Aldrich) antibodies. Peroxidase-conjugated secondary antibodies were purchased from Amersham ${ }^{\text {TM }}$.

\section{Statistical analysis}

Results are expressed as means \pm s.d. from an appropriate number of experiments. Differences were analyzed by ANOVA test using GraphPad Prism v.4.0 for Windows (GraphPad Software, San Diego, CA, USA, www. graphpad.com) and the threshold for statistical significance was set at 0.05. P-values are displayed on the graphs using a single asterisk for significances ranging from 0.05 to 0.01 , two asterisks for values between 0.01 and 0.001 and three asterisks for values below 0.001 .

\section{Results}

\section{Bio-nFeR nanomicelles}

To increase fenretinide bioavailability a new nanomicellar system was prepared by dissolving phosphatidylcholine and glyceryl tributyrate in alkaline ethanol in the presence of the drug. The alkaline solvent, providing ionization of the fenretinide phenolic hydroxyl allowed formation of ion pairs with the choline moiety of the phosphatidylcholine as a counterion. Subsequent evaporation of ethanol provided a semisolid residue containing the fenretinide - phosphatidylcholine ion pairs dispersed in the phospholipid excess-glyceryl tributyrate mixture (Fig. 1a). The dissolution in water of the evaporated residue provided spherical micelles as shown in Fig. 1 B reporting optical microscopy images obtained by dissolution of $100 \mathrm{mg} / \mathrm{ml}$ of the evaporated residue before filtration. Indeed, the thermodynamic stability of the fenretinide-phosphatidylcholine ion pair is the driving force for the formation of micelles characterized by a hydrophobic inner core containing fenretinide and an hydrophilic shell formed by the spontaneous assembling of the phospholipid molecules in water where the ion bridge stabilizes the core-shell interface [24].

As a comparison the same preparative procedure was followed by using pure ethanol instead of alkaline ethanol to prevent fenretinide ionization and ion pair formation. Fluorescence microscopy images, obtained by exploiting the spontaneous auto-fluorescence of fenretinide, showed spherical drug localizations inside the micelles prepared by fenretinide ionization, indicating the drug presence in the micellar inner cores in accordance with fenretinide-phosphatidylcholine ion pair formation. Without ionization, on the contrary, concentric drug localizations were obtained, suggestive of liposome-like structures with drug entrapment in the lipophilic phospholipid bilayers (Fig. 1c). The NCI-FeR images showed coarse aggregates related to lack of drug solubilization (Fig. 1b). Dynamic light scattering measures of Bio-nFeR indicated mean sizes in the nanometric scale, good dimensional polydispersity and a negative zeta potential (Fig. 1d). The solubilization ability of Bio-nFeR towards fenretinide, evaluated as the increase in drug concentration with the increase in nanomicelle concentration, was characterized by a linear trend indicative of a micellar-mode of drug solubilization (Fig. 1e). In micellar solubilization the linear trend persists until the micelle concentration become so high to cause self-aggregation and destabilization. In our study the linear trend lasted up to the maximum nanomicelle concentration evaluated $(300 \mathrm{mg} / \mathrm{ml})$ providing 25.41 $\mathrm{mg} / \mathrm{ml}$ fenretinide solubilization, much higher than the aqueous solubility of the pure drug $\left(1.7110^{-3} \mathrm{mg} / \mathrm{ml}\right)$.

The release of fenretinide from the nanomicelles was very low either at $\mathrm{pH} 1.2$ and $\mathrm{pH} 6.8$ being 8.2 and $21.19 \%$ respectively after $72 \mathrm{~h}$. In the presence of sodium taurocholate, on the contrary, the release was significantly improved being $48.45 \%$ at $72 \mathrm{~h}$ (Fig. 1f). This indicated stability of the Bio-nFeR nanomicelles towards drug leakage at the different $\mathrm{pH}$ of the gastrointestinal environment and their ability to promote intestinal lymphatic transport of fenretinide by the bile activated pathway of lipid absorption [5].

\section{Pharmacokinetic studies of bio-nFeR after acute and repeated administration}

As shown in Fig. 2a, after oral administration of different doses of Bio-nFeR to mice, 4-HPR achieves plasma Cmax between 2 and $4 \mathrm{~h}$, it is distributed rapidly and eliminated with half-life of about $7 \mathrm{~h}$, warranting a drug plasma exposure up to $48 \mathrm{~h}$ at each dose investigated. 

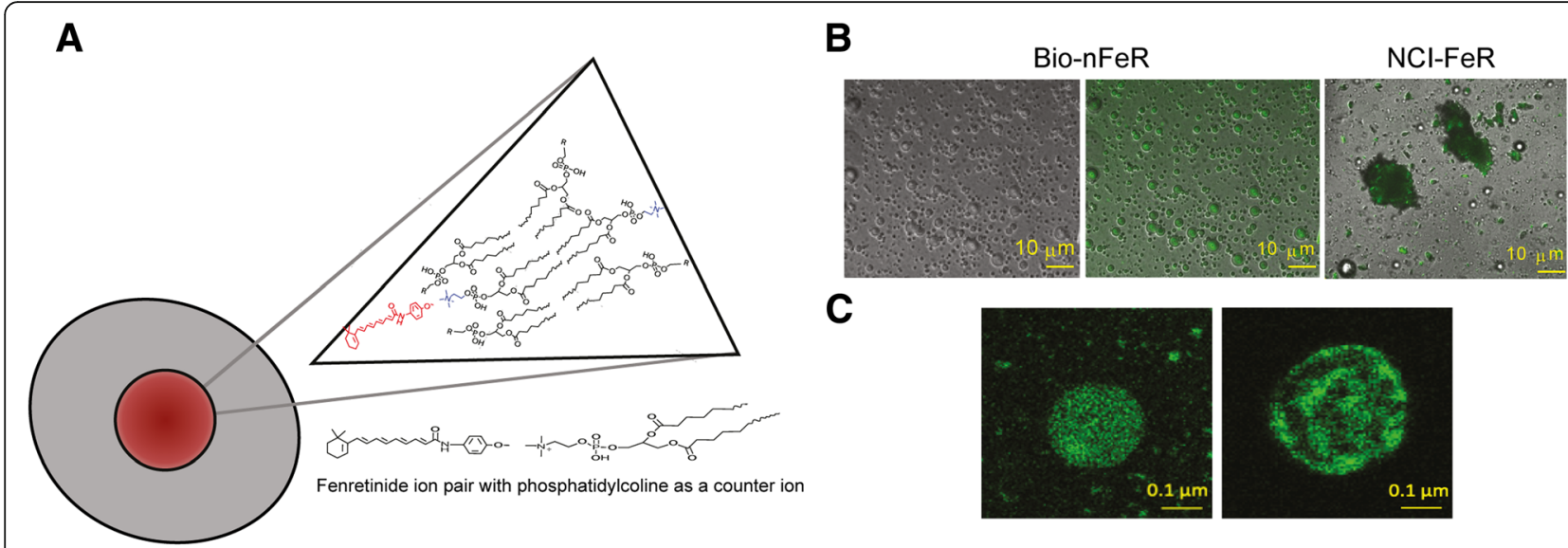

C

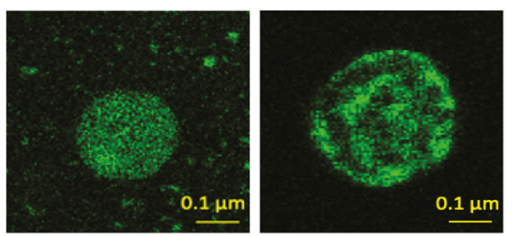

D

\begin{tabular}{|c|c|c|c|c|}
\hline Mean Size $(\mathrm{nm})$ & Polydispersity & Zeta Potential & Encapsulation Efficiency \% & Drug Loading Capacity \% (W:W) \\
\hline $300.5 \pm 11.5$ & $0.2306 \pm 0.016$ & $43.1 \pm 1.8$ & $91.5 \pm 3.2$ & $8.72 \pm 0.7$ \\
\hline
\end{tabular}

E

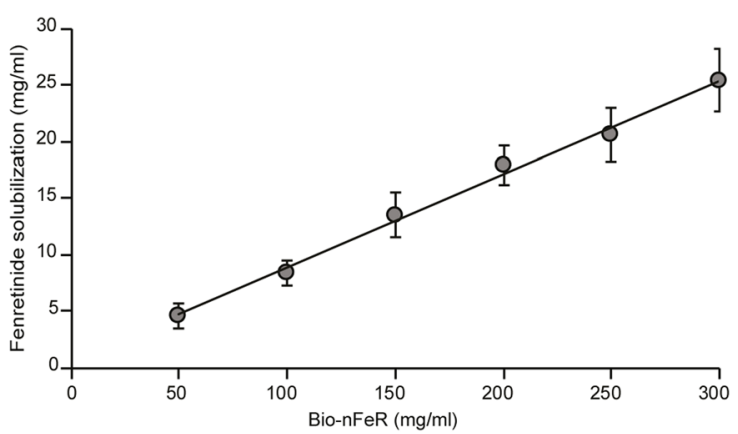

$\mathbf{F}$

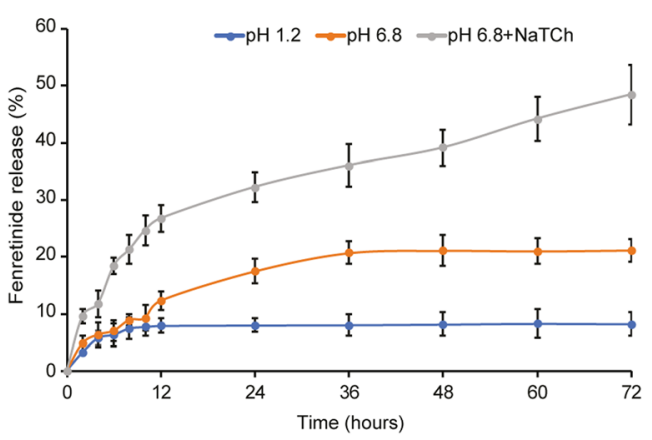

Fig. 1 Illustration of the Bio-nFeR biochemical structure and fluorescence microscopic image of drug-containing micelles. a Schematic representation of micelles composed of phospholipids in the outer layer and fenretinide in the core. $\mathbf{b}$ Aqueous dispersions of Bio-nFeR and $\mathrm{NCl}$ FeR formulations. (Left) Optical microscopy image of Bio-nFeR micelles in water. (Middle) Confocal image of Bio-nFeR (the same as in the left) showing the presence of autofluorescent fenretinide within micelles. (Right) Confocal image of NCl-FeR in water forming large aggregates of insoluble material (Images magnification is $60 \times$ and $10 \mu \mathrm{m}$ scale bar is reported). c Confocal laser scanning images of Bio-nFeR micelles obtained by fenretinide ionization (left) and micelles obtained by the same procedure but without fenretinide ionization (Images magnification is $60 \times$ and $0.1 \mu \mathrm{m}$ scale bar is reported). $\mathbf{d}$ Physico-chemical features of Bio-nFeR micelles. e Solubilization ability of Bio-nFeR towards fenretinide f) In vitro release of fenretinide from Bio-nFeR micelles in $\mathrm{HCl}$ solution ( $\mathrm{pH} 1.2$ ), phosphate buffer solution (pH 6.8) and phosphate buffer solution (pH 6.8) containing sodium taurocholate $(3.0 \mathrm{mM})$

The main derived pharmacokinetic parameters are listed in Table 1: Cmax and AUC increased proportionally with the doses, indicating that the pharmacokinetic of Bio-nFeR is not dose dependent in the range of doses studied as shown in Fig. 2b that reports the correlations between dose vs Cmax and vs AUC. These results suggest that drug dose could be increased further to reach superior plasma exposure, eventually enhancing the therapeutic effect.

Following the pharmacokinetic study of different doses as acute treatment, pharmacokinetic was investigated in an extensive comparative study, at therapeutic doses of Bio-nFeR and NCI-FeR. The two schedules of chronic administration were well tolerated in mice. No body weight loss or impaired liver function, were observed in treated- versus control-mice as carried out evaluating the hepatic enzymes levels (Additional file 1: Table S1). The schedules used are highly compatible with in vivo activity studies. $150 \mathrm{mg} / \mathrm{Kg}$ of Bio-nFeR and NCI-FeR were given as single administration (formally day 1 ) or for 2 weeks of daily administration (12 days on, 2 days off) and the plasma concentration-versus-time profiles of 4-HPR is shown in Fig. 2c. From a visual inspection of the curves and as indicated by the parameters listed in the Table 2, it can be seen that the exposure to 4-HPR is 1.3 and 1.6 times higher with oral Bio-nFeR than with 

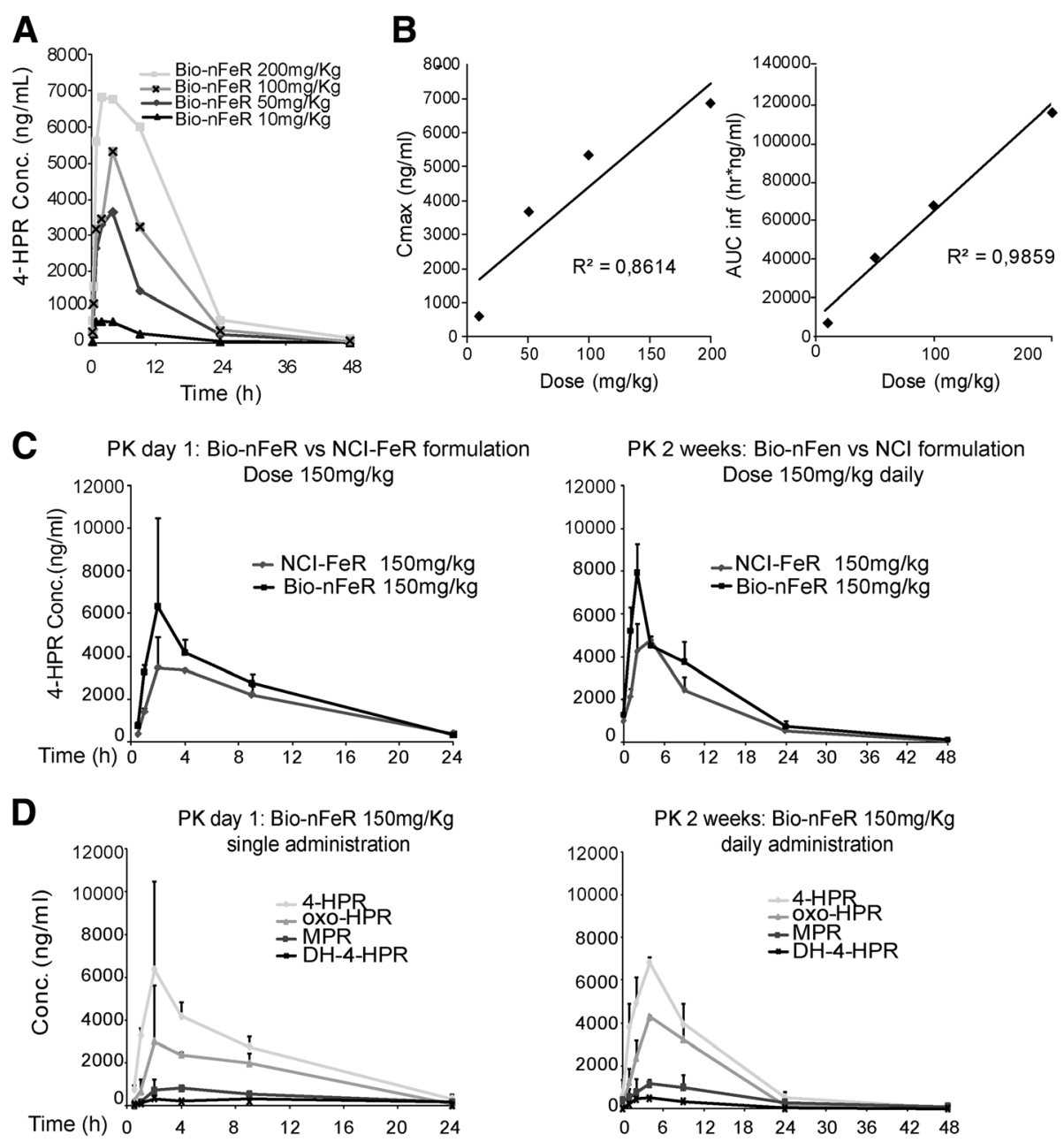

Fig. 2 (A-B) Plasma pharmacokinetic profiles of fenretinide after single oral administration of different Bio-nFeR doses. a Comparison of the fenretinide plasma concentration decay curves after administration of the indicated Bio-nFeR oral doses. $\mathbf{b}$ Cmax and AUC of fenretinide as function of the administered dose of Bio-nFeR. c-d Pharmacokinetic profile in acute and chronic administration of Bio-nFeR compared to NCI-FeR. c Pharmacokinetic profile of oral Bio-nFeR in comparison with fenretinide $\mathrm{NCl}$ formulation after acute treatment (left) or 2 weeks of chronic treatment (right). $\mathbf{d}$ Pharmacokinetic profile of oral Bio-nFeR and its metabolites as single administration or chronic treatment and its metabolites in the same samples as in C

Table 1 Bio-nFeR pharmacokinetic parameters

\begin{tabular}{lllll}
\hline Bio-nFeR pharmacokinetic & parameters & & \\
\hline Bio-nFer Dose $(\mathrm{mg} / \mathrm{kg})$ & 10 & 50 & 100 & 200 \\
Cmax $(\mathrm{ng} / \mathrm{ml})$ & 600.9 & 3654.7 & 5340.9 & 6837.8 \\
Tmax (hr) & 2 & 4 & 4 & 2 \\
AUC 0-last $\left(\mathrm{hr}{ }^{*} \mathrm{ng} / \mathrm{ml}\right)$ & 6745.4 & 39156.9 & 66323.6 & 112957.1 \\
AUC inf $\left(\mathrm{hr}^{*} \mathrm{ng} / \mathrm{ml}\right)$ & 6812.1 & 39513.7 & 66751.7 & 114227.6 \\
HL (hr) & 7.48 & 7.32 & 6.49 & 7.16 \\
\hline
\end{tabular}

Pharmacokinetic parameters analysis in mice after single oral treatment of 4 doses of Bio-nFeR: 10, 50, 100 and $200 \mathrm{mg} / \mathrm{kg}$. Cmax: maximum plasma concentration achieved after drug administration; Tmax: time until Cmax is reached; AUC last: experimental area under the concentration-time curve from time 0 to the last experimental point measured (Clast); AUC inf: AUC calculated from 0 to Clast added of the extrapolated portion of the AUC calculated by: $\mathrm{AUCz}=\mathrm{Clast} / \mathrm{ke}$; HL: plasma half-life of the terminal phase calculated by: $\mathrm{HL}=0.693 / \mathrm{ke}$. oral NCI-FeR after the first treatment and after the repeated treatments, respectively.

In details, following the administrations of Bio-nFeR, 4-HPR reaches Cmax between 2 and $4 \mathrm{~h}$, it is distributed rapidly and eliminated with a half life (HL) of about 5-6 $\mathrm{h}$ achieving adequate plasma exposure up to $48 \mathrm{~h}$ after the last administration. On day one, mean Cmax and AUC $_{0-24 \mathrm{~h}}$ were $6350 \mathrm{ng} / \mathrm{mL}$ and $56,881 \mathrm{ng} / \mathrm{mL}^{*} \mathrm{~h}$ and after the repeated treatment $6813 \mathrm{ng} / \mathrm{mL}$ and $78,608 \mathrm{ng} /$ $\mathrm{mL} \% \mathrm{~h}$. The last value indicates an increase of $40 \%$ of the plasmatic exposure of 4-HPR after chronic administration of Bio-nFeR in comparison with day one.

$\mathrm{R}^{*}=\mathrm{AUC}_{\text {inf }}$ ratio of repeated treatments vs acute treatment normalized per dose.

The elimination of fenretinide takes place mainly by metabolic process with formation of 4-oxo-4-HPR and 
Table 2 Pharmacokinetic comparison of Bio-nFeR and NCl-Fer in single versus chronic treatment

\begin{tabular}{|c|c|c|c|c|}
\hline \multirow[b]{3}{*}{ Parameters } & \multicolumn{4}{|c|}{ 4-HPR dose 150 mg/Kg } \\
\hline & \multicolumn{2}{|c|}{ Single acute treatment } & \multicolumn{2}{|c|}{2 weeks of treatment daily } \\
\hline & $\mathrm{NCl}-\mathrm{FeR}$ & Bio-nFeR & $\mathrm{NCl}-\mathrm{FeR}$ & Bio-nFeR \\
\hline Cmax (ng/ml) SD & $3456.8 \pm 1453.3$ & $6350 \pm 4153.9$ & $4306.4 \pm 1150.7$ & $6813.3 \pm 1200.9$ \\
\hline Tmax (hr) & 2 & 2 & 2 & 4 \\
\hline AUC inf (hr*ng/ml) & 46873.8 & 59371 & 53554.7 & 85378.7 \\
\hline AUC 24 h (hr.*ng/ml) & 43257.7 & 56880.7 & 45436.7 & 78608.3 \\
\hline $\mathrm{HL}(\mathrm{hr})$ & 6.4 & 5.3 & 8.3 & 5.7 \\
\hline$R^{*}$ & 1.3 & & 1.6 & \\
\hline
\end{tabular}

Pharmacokinetic parameters determination in mice after single oral treatment of Bio-nFeR at dose of $150 \mathrm{mg} / \mathrm{kg}$ or after $2 \mathrm{weeks}$ treatment, in comparison to the $\mathrm{NCl}-\mathrm{FeR}$ formulation

MPR and via fecal excretion of the unmodified 4-HPR and metabolites.

As it can be seen in Additional file 1: Figure S1A reporting the plasma concentration-time profiles of the metabolites obtained in the study of multiple doses, 4-oxo-4-HPR and MPR in comparison to 4-HPR, amounted to about 50 and 15\% after Bio-nFeR administration. Similar results were obtained for NCI-FeR administrations in comparative experiments of the two formulations (Additional file 1: Figure S1B). The main pharmacokinetic parameters obtained for Bio-nFer metabolites are reported in Additional file 1: Table S2A, comparison of single vs chronic BionFer treatment in Fig. 2d and comparison of Bio-nFer vs $\mathrm{NCI}$ in Additional file 1: Table S2B.

A similar metabolic picture was seen during chronic administrations being the metabolites measurable in plasma 1 hour after administration and with maximal exposure at $2-4 \mathrm{~h}$ (Fig. 2d). The metabolites disappear with the same half-life of the parent compound (Additional file 1: Table S3). As seen in the acute study, the relative percent amount versus 4-HPR of 4-oxo-4-HPR, known to be 2-4-fold more cytotoxic than 4-HPR, and of the inactive metabolite MPR, after single and repeated treatment corresponded to about 50 and 15\%, respectively. In addiction we detected the presence of a third metabolite, DH-4-HPR, previously described by Cooper et al. [4] that amounted to about $5-7 \%$ of the parental drug. As last consideration on metabolites formation, no significant differences were recorded in their plasma AUC between day 1 and day 14 (the metabolites AUCs increase, but reflect in a proportional way the increase of AUC of the parent drug), thus indicating that no phenomena of saturation or induction of the metabolism are present after the chronic treatment (Additional file 1: Table S3).

The fecal excretion of the parent drug and metabolites is reported in Additional file 1: Table S4. After both studies, the acute treatment at multiple doses and the chronic one, the proportion of Bio-nFeR and NCI-FeR eliminated in faeces as unmodified 4-HPR is comprised in the ranges $2-9 \%$, for 4-oxo-4-HPR 4-
$33 \%$, for DH-4-HPR $1-12 \%$ and less than $0.1 \%$ for MPR.

The amount of dose recovered in urine is negligible $(\leq 0.002 \%)$ in line with the low polarity of fenretinide and metabolites (data not shown).

\section{Bio-nFeR exerts increased toxicity against lung CSC in vitro and exhibits improved plasma exposure}

With the aim to determine whether Bio-nFeR was endowed with valuable antitumour activity, its ability to affect lung-CSC viability was evaluated in comparison with the standard oral capsule formulation of fenretinide (NCI-FeR). Five previously isolated and validated patientderived lung-CSC of different subtypes (Squamous Cell Carcinoma, Adenocarcinoma and Large cell Carcinoma) [44], were exposed to three different doses of Bio-nFeR or in parallel to NCI-FeR at the same concentrations. BionFeR exerted a substantial cytotoxic effect, against all lung-CSC examined even at low concentrations although with variable extent in different samples, while more than 95\% cells were killed at higher doses in all samples tested. In contrast, NCI-FeR determined a moderate (40-60\%) reduction in cell viability only at higher doses $(10 \mu \mathrm{M})$. More precisely, Bio-nFeR inhibited cell viability to a comparable extent than that determined by 10 fold higher concentration of NCI-FeR within the drug doses range tested, as visible in MTT assay-derived viability curve. Moreover, these finding was confirmed by the $\mathrm{IC}_{50}$ values calculated through a comparative dose response experiment performed on lung CSC that evidenced a 10 fold difference in IC50 with Bio-nFeR $\mathrm{IC}_{50}=0.72 \mu \mathrm{M}$ and NCIFeR $\mathrm{IC}_{50}=9 \mu \mathrm{M}$ (Fig. 3a and Additional file 1: Figure S2).

We next examined the antitumour activity of Bio-nFeR in lung-CSC derived xenografts obtained in NSG immunodeficient mice, in comparison with the standard NCIFeR formulation. While NCI-FeR administration (50 mg/ $\mathrm{kg}$ ) did not substantially affect tumour growth rates compared to tumours of untreated mice, the same dose of Bio-nFeR determined a significant inhibition of tumour growth (Fig. 3b left). Importantly, no signs of 


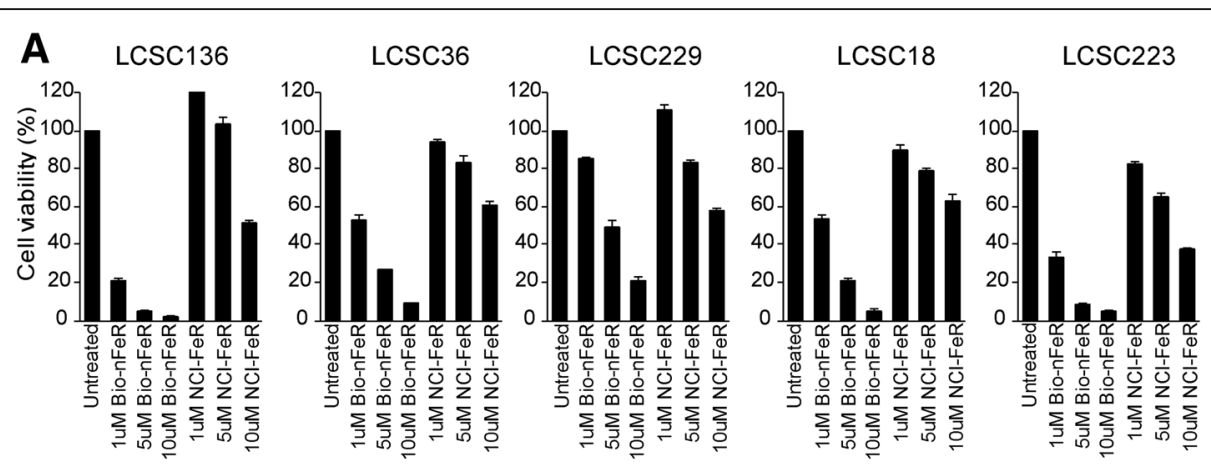

B

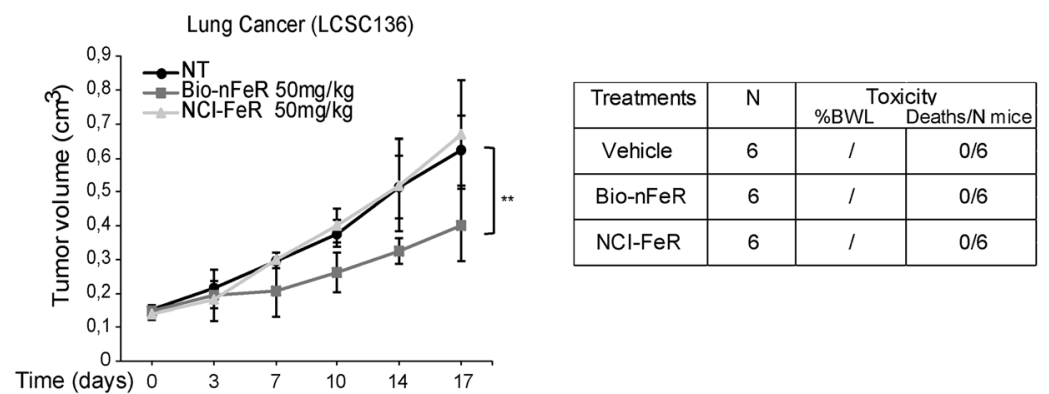

Fig. 3 (a) Cytotoxic activity of Bionanofenretinide (Bio-nFeR) in comparison with standard formulation fenretinide (NCI-FeR) in lung CSC. Lung CSC were exposed to the indicated drug doses and cell viability was evaluated by CellTiter-Glo after $72 \mathrm{~h}$ and indicated as percentage versus control cells. b Bio-nFeR and NCI-FeR antitumour activity in lung CSC-derived xenografts. (left) Growth curves of lung CSC-derived xenografts in control mice or mice treated with Bio-nFeR or NCl-FeR at $50 \mathrm{mg} / \mathrm{kg}$ dose for the indicated times. Lung CSC sample \#136 was used for xenograft generation. Mean \pm S.D. of three independent experiments is shown. ${ }^{*} P<0.01$. (right) Table of drug-induced systemic toxicity in the three groups of mice indicated as percentage of body weight loss (BWL) or number of deaths/total number of mice

systemic toxicity (Fig. 3b right) were noticed suggesting that increased Bio-nFeR doses could be safely explored for future experiments, with the aim to further enhance efficacy.

\section{Bio-nFeR displays broad antitumour activity in vitro and} in vivo, is well tolerated and reaches elevated and pharmacologically active intra-tumour concentrations Given the marked antitumour activity of Bio-nFeR against lung cancer cells in vitro and in vivo and the enhanced systemic exposure compared to the reference fenretinide formulation, we next extended the evaluation of its cytotoxic activity in a wide range of concentrations against lung, colon cancer, melanoma, sarcoma and glioblastoma CSC. Three days exposure to Bio-nFeR determined a marked inhibition of CSC viability in the majority of CSC samples of each tumour type tested (Fig. 4a). Generally, while glioblastoma and sarcoma-CSC lines resulted more resistant to Bio-nFer treatment, a high fraction of lung, colon and melanoma CSC lines displayed promising responsiveness to Bio-nFeR, prompting subsequent in vivo studies to assess the drug antitumour activity in the corresponding xenograft models. Subcutaneous CSC-derived lung, melanoma and colon tumours were generated in immunodeficient mice and Bio-nFeR was orally administered for 3 weeks at 100 (lung cancer and melanoma) or 150 (colon cancer) $\mathrm{mg} / \mathrm{kg}$, based on the absence of toxicity demonstrated in the previous experiment (Fig. 3b), where lower doses of drug were used. Tumours of treated mice showed a prominent reduction of the tumour growth rate, compared to control tumours (Fig. 4b). Moreover, the drug displayed high tolerability, as no systemic toxicity was observed, except for a minor (8\%) weight loss in the higher-dose mice group $(150 \mathrm{mg} / \mathrm{kg})$, in absence of other visible signs of toxicity (Fig. 4b bottom panels). The lack of mice toxicity, together with acceptable patient tolerability observed when 10 times higher doses have been administered in clinical trials, suggests that the maximum tolerated dose has not been achieved under these experimental conditions and can be safely escalated to further enhance drug amount at the tumour site and therapeutic efficacy [13].

In line with its antitumour efficacy, described above, the parallel pharmacokinetic study showed that fenretinide reached highly encouraging concentrations in plasma (Fig. 4c), higher than that reached in plasma of patients treated with equivalent doses $\left(300 \mathrm{mg} / \mathrm{m}^{2}\right.$ is the equivalent dose corresponding to $100 \mathrm{mg} / \mathrm{kg}$ in mice) of the standard drug or of other improved drug formulations in clinical trials, both as single or multiple administrations [20,27].

Importantly, as measured here for the first time in tumour models, the drug showed pharmacologically 
A
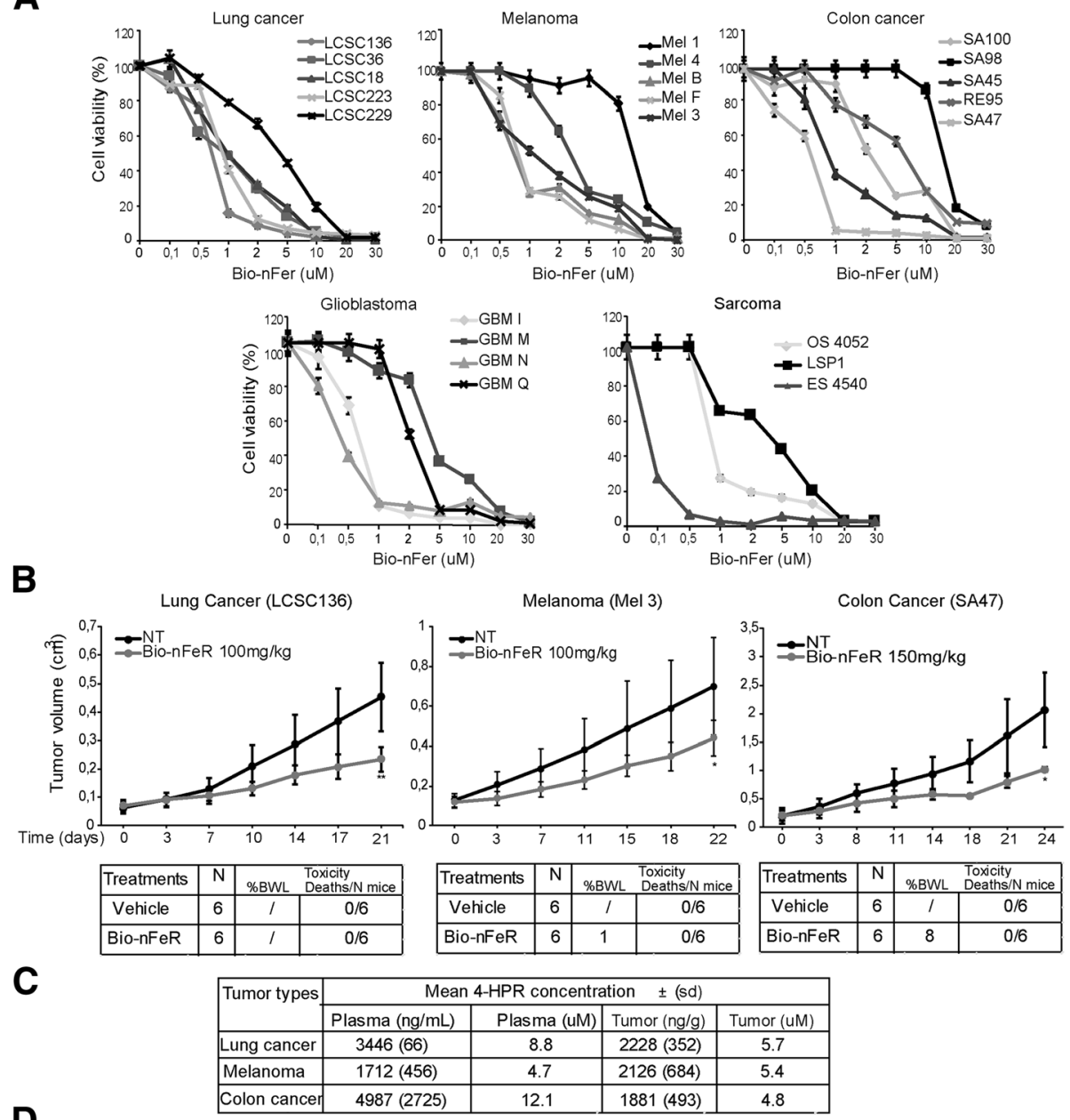

D
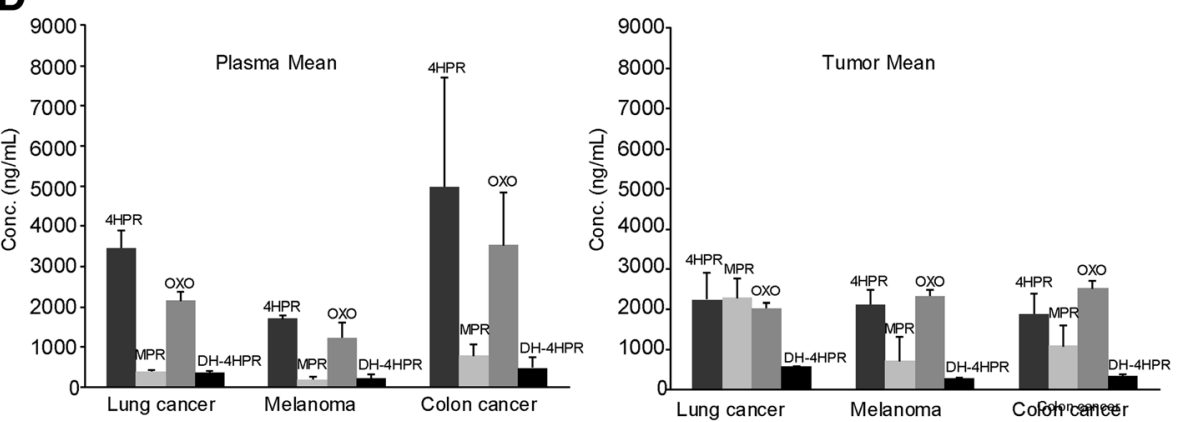

Fig. 4 (a) Cytotoxic activity of different doses of Bio-nFeR in lung cancer, melanoma, colon cancer, glioblastoma and sarcoma CSC in vitro. The indicated CSC were exposed to 0.1, 0.5, 1, 2, 5, 10, 20 and $30 \mu \mathrm{M}$ drug doses and cell viability was evaluated by Cell Titer-Glo after $72 \mathrm{~h}$ and indicated as percentage versus control cells. b Bio-nFeR antitumour activity in lung cancer, melanoma and colon cancer CSC-derived xenografts. (Upper panels) Growth curves of CSC-derived xenografts in control mice or mice treated with Bio-nFeR at 100 (lung cancer and melanoma) or 150 (colon cancer) $\mathrm{mg} / \mathrm{kg}$ dose for the indicated times. Mean \pm S.D. of three independent experiments is shown. ${ }^{*} P<0.05{ }^{* *} P<0.01$. (lower panels) Table of drug-induced systemic toxicity in the three groups of mice indicated as percentage of body weight loss (BWL) or number of deaths/total number of mice. c Fenretinide levels in plasma and tumours of the same samples as in B. Fenretinide concentration in plasma and tumours is expressed in $\mathrm{ng} / \mathrm{ml}$ and the corresponding $\mu \mathrm{M}$ concentration, as indicated (tumour density is assumed as approximately =1). $\mathbf{d}$ Fenretinide metabolites levels in plasma and tumours of the same samples as in B-C. Fenretinide, 4HPR, OXO-4HPR and DH-4HPR concentration levels in the indicated plasma and tumours of the same samples as in C 
active intra-tumour concentration (ranging from $1.88 \mu \mathrm{g} / \mathrm{g}$ to $2.23 \mu \mathrm{g} / \mathrm{g}$, equivalent to $4.8-5.7 \mu \mathrm{M})$ at both drug doses analyzed (Fig. 4c). These concentrations were superior to those required for cytotoxicity in vitro in these cell lines (Fig. 4a).

In the tumours analyzed, the three main metabolites of 4-HPR already seen in plasma were detected (Fig. 4d). The most abundant was 4-oxo-4-HPR, the active one, that achieved comparable tumour concentrations in the three different models. These concentrations were in the same range of those of the parent drug, but since this metabolite is 2-4 fold more active than 4-HPR, we can speculate that the in vivo antitumour activity is mainly due to its conspicuous presence (Additional file 1: Table S5).

\section{Bio-nFeR antitumour activity is associated with reduced} tumour cell proliferation, apoptosis induction, modulation of lipid metabolism and decrease of CSC features

To dissect the molecular effects of Bio-nFeR treatment in vivo, control and treated tumour xenografts were analyzed for their proliferative index and extent of cell death induction. As clearly visible in confocal images of tumour tissues slides in Fig. 5a, the expression of KI-67 was strongly reduced in all treated tumours. Moreover, tumours treated with Bio-nFeR at both doses of 100 and $150 \mathrm{mg} / \mathrm{kg}$ displayed a markedly increased fraction of TUNEL-positive cells in comparison with controls (Fig. 5b). Thus, Bio-nFeR induced a marked inhibition of tumour cell proliferation associated with apoptosis induction, in line with previous reports $[2,8]$.

Previous reports showed that fenretinide-mediated cytotoxicity is associated with dysregulation of lipid metabolism. Specifically, fenretinide is able to increase the intracellular levels of dihydroceramides species by targeting the dihydroceramide desaturase [16, 29, 38, 51]. Liquid chromatography-mass spectrometry (LC-MS), showed that Bio-nFeR treatment induced a marked increase of dihydroceramide and dihydrosphingolipids (glucosyldihydroceramide) species (Fig. 5c and Additional file 1: Figure S3). This effect is more evident for dihydroceramides species with 18 and 16 fatty-acid chains. Conversely, saturated ceramides levels were not affected by Bio-nFeR treatment. In concomitance with the accumulation of long-chain dihydroceramide, we also observed a significant increase of the intracellular levels of sphinganine in Bio-nFeR-treated tumour xenografts (Fig. 5d). Based on our previous results, we next confirmed that xenografts derived from the specific CSC lines used were
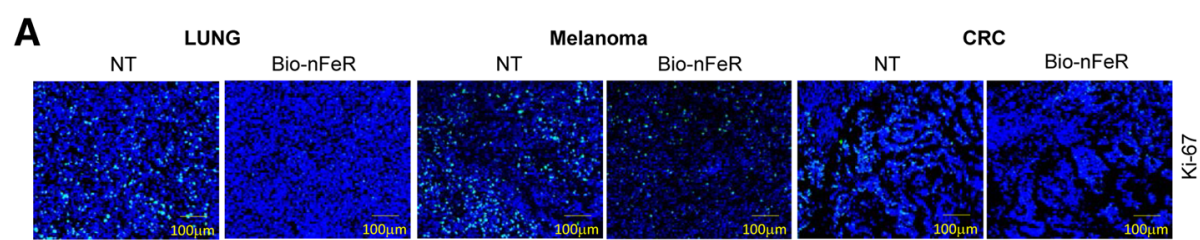

B

NT

Bio-nFeR

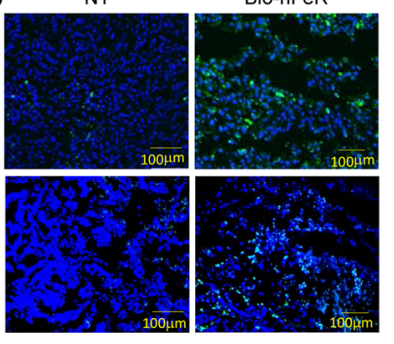

C

D
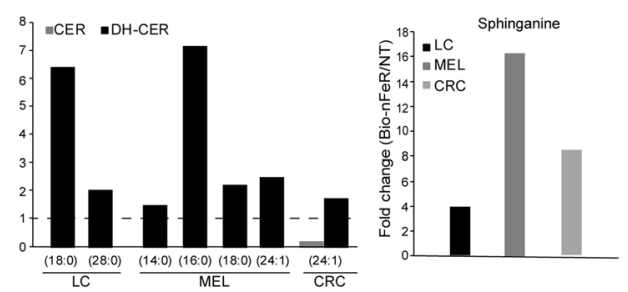

$\mathbf{E}$

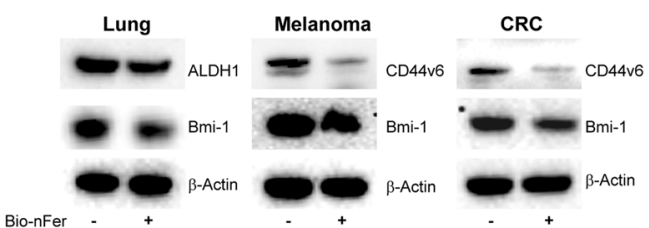

Fig. 5 Reduction of tumour cell proliferation, apoptosis induction and modulation of lipid metabolism by Bio-nFeR. a Confocal images of Kl-67 immunofluorescence of tissue slides from control or Bio-nFeR treated lung cancer, melanoma and colorectal (CRC) cancer xenografts. b TUNEL assay, showing the levels of apoptosis induction at both doses used (upper panels correspond to $100 \mathrm{mg} / \mathrm{kg}$-treated lung cancer xenografts and lower panels to $150 \mathrm{mg} / \mathrm{kg}$-treated colon cancer xenografts). $\mathbf{c}$ Individual dihydroceramides composition of lung (LC), melanoma (MEL) and colorectal (CRC) tumours treated with Bio-nFeR at single 100-150 mg/Kg administration were identified by acyl chain, normalized to control and plotted as fold change. $\mathbf{d}$ The amount of sphinganine in the same samples as in A and B, normalized to control and plotted as fold change. e Immunoblot analysis of CSC antigens $\mathrm{ALDH1}, \mathrm{CD} 44 \mathrm{~V} 6$ and Bmi-1 in the indicated control $(-)$ or Bio-nFeR treated $(+)$ xenografts. $\beta$-actin blot was used for equal loading control 
heterogeneous and composed by a minor sub-population of CSC within a majority of differentiated tumour cells $[42,47,50]$. In fact, flow cytometry analysis for CSC specific markers confirmed that while aldefluor+ (lung) or CD44V6+ (melanoma and colon) cells constituted a large fraction of the CSC culture population in vitro as expected for a CSC-enriched population, this fraction dramatically decreased in the corresponding xenografts (Additional file 1: Figure S4A). Thus, to determine whether Bio-nFeR treatment was effective against CSC within the tumour, we analyzed the CSC features of control or Bio-nFeR treated tumour xenografts, based on the expression of CSC-restricted antigens suitable for each tumour type [7, 9, 21, 41, $42,44,46,47,50]$. We found that xenografts derived from mice treated with Bio-nFeR displayed decreased expression of CSC-specific antigens compared to control tumour, demonstrating that drug treatment determined a preferential CSC targeting within the tumour. Thus, Bio-nFeR tumours displayed decreased CSC features, implying decreased aggressiveness as compared with control tumour (Fig. 5e and Additional file 1: Figure S4B).

As we observed that Bio-nFeR was superior than NCI$\mathrm{FeR}$ at $50 \mathrm{mg} / \mathrm{kg}$ dose (Fig. 3) in terms of anti tumour activity and that increased HPR concentration was detected in plasma of Bio-nFeR treated mice compared to NCI-FeR treated animals at higher therapeutic doses (Fig. 2), we next evaluated whether at the dose of 100 $\mathrm{mg} / \mathrm{kg}$, therapeutically active for Bio-nFeR, NCI-FeR formulation could reach therapeutic drug levels within tumour. In a comparative study, in which tumour-bearing mice were treated with the two alternative drug formulations at $100 \mathrm{mg} / \mathrm{kg}$ dose, Bio-nFeR confirmed superior antitumour activity compared to NCI-FeR (Additional file 1: Figure S5), as well as higher plasma bioavailability (Fig. 6a). Moreover, in line with these results, Bio-nFeR treatment determined much higher intra tumour concentration of 4-HPR and its active metabolite 4-oxo-4-HPR, compared to that reached following NCI-FeR administration (Fig. 6), demonstrating the higher tropism of Bio-nFeR also at the tumour level, in line with its superior anti tumour activity.

\section{Discussion}

Cellular and molecular heterogeneity of tumours represents an obstacle for the achievement of long-term therapeutic efficacy. Cell fractions endowed with therapy-resistant stem cell-associated features are represented by CSC, which are themselves a heterogeneous population characterized by epigenetic and functional traits rather than specific molecular alterations [6]. Thus, tumour diversity needs to be accurately considered, to become an option for cancer therapy. For instance, specific CSC-properties can be targeted to achieve durable effects and the efficacy of molecularly-targeted compounds needs to be validated in the CSC compartment to assess its long-term therapeutic benefit. Furthermore, another issue is the necessity to reach therapeutic drug concentrations at the tumour site to increase drug activity against the more resistant CSC while reducing systemic toxicity. This aim can be accomplished through targeted-delivery of drugs to the tumour site or through appropriate drug modifications favoring accumulation at the target site, limiting drug degradation in the blood or increasing its solubility in body fluids and in tumour microenvironment.

Here, we explored the activity of a promising anti-tumour agent, fenretinide, against CSC of different tumours upon enhancing its antitumour activity through a nanocarrierbased approach. Fenretinide has been previously proved to be a promising anticancer agent with ability to affect CSCrelated properties and viability of cell lines of different tumours including medulloblastoma, melanoma, breast and myeloma cell lines [1, 10, 33, 53]. In contrast, fenretinide activity in vivo is severely hampered by its hydrophobic character which restrains its bioavailability and prevents the achievement of therapeutic drug levels in the body compartments. Thus, due to fenretinide potential cytotoxic activity against aggressive tumour cells and in view of its proved negligible systemic toxicity, drug development has been fostered through drug formulations aimed at improving its solubility and bioavailability. Encouraging results have been obtained in this direction, including a fenretinide-cyclodextrin complex developed in our laboratory which, however, required intravenous administration [36] $([4,8,20]$; B. J. [27, 32, 37]).

We developed a new nano-micellar fenretinide formulation called bionanofenretinide (Bio-nFeR), based on ion pair formation between fenretinide and phosphatidylcholine. Such pairing triggers the spontaneous formation in water of micelles, containing a fenretinide inner core and an hydrophilic shell formed by the spontaneous assembling of the phospholipid molecules. The nanomicelles were designed to raise fenretinide solubility and bioavailability by oral administration and provide drug levels in the body compartments suitable to elicit a therapeutic response. To assess the therapeutic potential of the Bio-nFeR formulation we compared its activity in vitro and in tumour xenografts with that of the standard formulation made of soft gelatin capsules containing fenretinide in corn oil and polysorbate, which is currently available at the National Cancer Institute (NCI-FeR) and has been largely tested in clinical trials [13]. Bio-nFeR displayed encouraging cytotoxic activity against all lungCSC tested and its activity was superior than the standard NCI-FeR, with more than 10 fold difference in the $\mathrm{IC}_{50}$ in most cases (Fig. 2a and Additional file 1: Figure S2). Enhanced antitumour activity was observed in vivo, as Bio-nFeR proved to be far more effective in the 
A

\begin{tabular}{|c|c|c|c|c|c|c|c|}
\hline \multicolumn{8}{|c|}{ Mean ( $\pm S D)$ 4-HPR concentration } \\
\hline \multicolumn{2}{|c|}{$\mathrm{NCl}-\mathrm{FeR}$} & \multicolumn{2}{|c|}{ Bio $-n F e R$} & \multicolumn{2}{|c|}{$\mathrm{NCl}-\mathrm{FeR}$} & \multicolumn{2}{|c|}{ Bio $-n F e R$} \\
\hline \multicolumn{4}{|c|}{ Plasma } & \multicolumn{4}{|c|}{ Tumor } \\
\hline$(\mathrm{ng} / \mathrm{ml})$ & $(\mu \mathrm{M})$ & $(\mathrm{ng} / \mathrm{ml})$ & $(\mu \mathrm{M})$ & $(\mathrm{ng} / \mathrm{g})$ & $(\mu \mathrm{M})$ & $(\mathrm{ng} / \mathrm{g})$ & $(\mu \mathrm{M})$ \\
\hline $\begin{array}{l}408.0 \\
(98.9) \\
\end{array}$ & 1.0 & $\begin{array}{c}3616.7 \\
(1981.2)\end{array}$ & 9.2 & $\begin{array}{c}586.0 \\
(257.0)\end{array}$ & 1.5 & $\begin{array}{l}3771.3 \\
(646.5)\end{array}$ & 9.6 \\
\hline
\end{tabular}

B
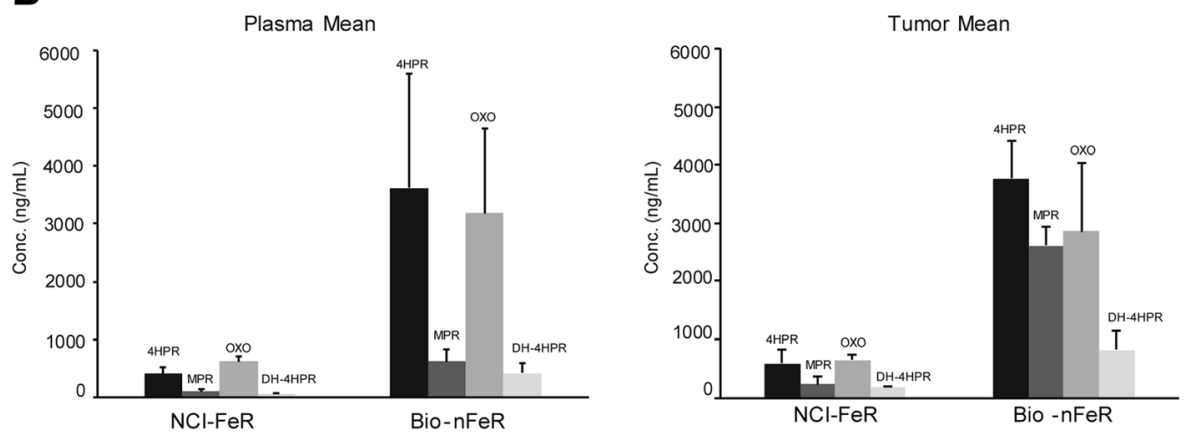

Fig. 6 Comparison of fenretinide levels in blood and tumours of mice treated with standard NCI-FeR or Bio-nFeR formulations. a Fenretinide concentration ( \pm standard deviation) in plasma (expressed in $\mathrm{ng} / \mathrm{ml}$ and the corresponding $\mu \mathrm{M}$ concentration are indicated) and tumours (in $\mathrm{ng} /$ $\mathrm{g}$ and the extrapolated $\mu \mathrm{M}$ concentration assuming tumour density as approximately $=1$ ) of mice after 3 weeks $100 \mathrm{mg} / \mathrm{kg}$ oral administration of each drug formulation. $\mathbf{b}$ Fenretinide metabolites levels in plasma and tumours of the same samples as in A. Fenretinide, 4HPR, OXO-4HPR and DH-4HPR concentration levels in the indicated plasma and tumours of the same samples as in A

growth inhibition of xenografts generated by lung-CSC (Fig. 4). Importantly, no signs of systemic toxicity were noticed, in line with the high tolerability described for patients treated with comparable doses of different formulations in clinical trials and suggesting that increased Bio-nFeR doses could be safely explored for future experiments, with the aim to further enhance its efficacy. In line with robust activity in vivo, Bio-nFeR displayed an improved pharmacokinetic profile than NCI-FeR. In fact, in contrast to NCI-FeR that showed a dose dependent pharmacokinetic curve, the pharmacokinetics of Bio-nFeR was not dose dependent and appeared linear in the doses range studied (Fig. 2c). These observations suggest that drug dose could be further increased to reach superior plasma exposure and enhance the therapeutic effect.

Besides lung cancer, Bio-nFeR cytotoxic activity was evaluated in a wide range of patient-derived CSC samples of different origin including melanoma, glioblastoma, colon cancer and sarcoma. In the context of a general marked efficacy, only a minority of CSC samples resulted less sensitive to Bio-nFeR suggesting that the drug is endowed with broad and prominent CSC-efficacy, even though biomarkers of response might be helpful to identify the responsive cells. The more promising drug activity was observed for lung, colon and melanoma tumour-derived CSC and the corresponding CSC-derived xenografts displayed high sensitivity to BionFeR treatment (Fig. 4). Based on the lack of toxicity observed in the first experiments drug doses were escalated up to $100-150 \mathrm{mg} / \mathrm{kg}$, determining improved efficacy with negligible side effects. Bio-nFeR antitumour activity was associated with reduced tumour cell proliferation, apoptosis induction and modulation of lipid metabolism at both Bio-nFer doses used (Fig. 5). The fenretinide doses used in mice experiments are comparable to the lower range doses used in drug escalation clinical trials using NCI-FeR or other fenretinide formulations, accordingly to the body surface area conversion method used to calculate the human equivalent doses (HED), as previously reported [39]. In fact, as $100 \mathrm{mg} / \mathrm{kg}$ (dose used in mice) is equivalent to $300 \mathrm{mg} / \mathrm{m} 2$, and up to 10 times higher doses were tolerated in clinical trial patients, it is likely that the drug dose of Bio-nFeR can be escalated further in absence of substantial side effects and the therapeutic efficacy correspondingly increased (the maximum dose used is pediatric cinical trial has been $4000 \mathrm{mg} / \mathrm{m} 2$ and displayed manageable toxicity) $[13,15]$.

Our pharmacokinetics studies showed that single Bio$\mathrm{nFeR}$ administration at $100 \mathrm{mg} / \mathrm{kg} /$ daily determined high plasma concentrations, higher than the therapeutic concentrations and superior to those found in other mice studies after both single or chronic administration of the standard fenretinide formulation (NCI-FeR) or with the more recently introduced oral powder lipid matrix formulation, called LYM-X-SORB matrix $(13.64 \mu \mathrm{M}$ plasma concentration was obtained after a single $100 \mathrm{mg} / \mathrm{kg}$ Bio- 
$\mathrm{nFeR}$ administration, versus $1.6 \mu \mathrm{M}$ reported for NCI-FeR and $5.1 \mu \mathrm{M}$ for LYM-X-SORB after repeated administrations of $120 \mathrm{mg} / \mathrm{kg}$ for 4.5 days) (Barry J. [26]).

Thus, the oral bioavailability has been found to be acceptable and reproducible in the doses range studied. Bio-nFeR has showed, at therapeutic doses of fenretinide, higher systemic exposure than that obtained with the reference NCI formulation, especially during the chronic pharmacokinetic study conducted after 1 or 2 weeks of daily administration. The high bioavailability contributed to reach unexpectedly high drug concentrations within tumour tissue in three different models tested (lung, colon and melanoma) after mice treatment with Bio-nFeR (Fig. 4c, d). In fact, the new formulation showed intratumour concentration of fenretinide pharmacologically active, superior to those required for cytotoxicity in vitro (Figs. 2a and 3). Finally, intratumour concentration of $4 \mathrm{HPR}$ obtained following Bio-nFeR treatment was much higher than that obtained with the standard NCI-FeR administered at the same dose. These results enforced the assumption that the increased antitumour efficacy of Bio-nFeR in vivo, compared to NCI$\mathrm{FeR}$, is the direct consequence of the improved bioavailability obtained with the new formulation that achieved higher drug concentration at tumour site.

\section{Conclusions}

In conclusion, with the aim to explore the possibility of fully exploit the therapeutic potential of fenretinide, we generated and tested, for the first time in in vitro and in vivo models, the anti-tumour activity of fenretinide nanoencapsulated in a new micellar system, Bio-nFeR, designed to improve its bioavailability and obtain effective anti-tumour plasma concentrations. Bio-nFeR therapeutic efficacy was superior to previous formulations and displayed negligible systemic toxicity at therapeutic doses. Our results propose Bio-nFeR as a CSC-effective innovative formulation of fenretinide with increased antitumour activity in vitro and in vivo due to improved drug solubility and bioavailability, that guarantees therapeutic drug exposure at tumour site in the absence of toxicity. Our results strongly encourage further preclinical-investigation on Bio-nFer in solid tumour CSC in the direction of clinical development.

\section{Additional file}

Additional file 1: Figure S1. Metabolites plasma concentrations (MPR and oxo-4-HPR) determined in mice after single oral treatment of 4 doses of Bio-nFeR: 10, 50, 100 and $200 \mathrm{mg} / \mathrm{Kg}$. The analysis was performed after 0, 24, 48 h. Figure S2. Evaluation of the 50\% inhibitory concentration (IC50) of BionFeR and $\mathrm{NCl}$-FeR formulation was done on a representative lung cancer stem cell line (LCSC). The cells were exposed to the indicated drug doses $(0-30 \mu \mathrm{M})$ and cell viability was evaluated by CellTiter-Glo after 72 h. Figure S3. A) Sphingomyelin-Ceramide (SM-CER) and Sphingomyelin- Dihydro-Ceramide (SM-DH-CER) content in lung (LC), melanoma (MEL) and colon (CRC) tumors treated with Bio-nFeR at single 100-150 mg/Kg administration were quantified through Ultra High Performance Liquid Chromatography (UHPLC), normalized to control and plotted as fold change. B) Quantification of Glucosyl-Ceramide (GLC-CER) and Glucosyl-Dihydro-Ceramide (GLC-DH-CER) in the same samples as in A, normalized to control and plotted as fold change. Figure S4. Cancer stem cell marker expression in CSC-culture conditions and in CSC-derived xenografts. A) Flow cytometry analysis of Aldefluor in (left panels) and CD44v6 (middle and right panels) in the indicated CSC and xenograft samples. B) Immunoblot analysis of CSC antigens C-Kit and SOX-2 in the indicated control $(-)$ or BionfeR treated (+) melanoma xenografts. $\beta$-actin blot was used for equal loading control. Figure S5. Growth curves of lung CSC-derived xenografts in control mice or mice treated with Bio$\mathrm{nFeR}$ or $\mathrm{NCl}-\mathrm{FeR}$ at $100 \mathrm{mg} / \mathrm{kg}$ dose for the indicated times. Mean \pm standard error is shown. ${ }^{*} P<0.05$. Table $\mathbf{S 1}$. Hepatic enzyme determination in mice after single administration or 2 weeks oral daily treatment at dose of $150 \mathrm{mg} / \mathrm{kg}$ of Bio-nFeR or NCIFeR formulation. AST: Aspartate transaminase; ALT: Alanine transaminase; Bil tot: Bilirubin total; Vehicle after $24 \mathrm{~h}$. Table S2. Quantification of 4-HPR and metabolites (oxo-4-HPR e MPR) determined in mice after oral Bio-nFeR single treatment of BNF: 10, 50, 100 and $200 \mathrm{mg} / \mathrm{Kg}$ (A) or Bio-nFer at dose of $200 \mathrm{mg} / \mathrm{kg}$ in comparison to the NCl-FeR formulation (B). Cmax: maximum plasma concentration achieved after drug administration; Tmax: time until Cmax is reached; AUC last: experimental area under the concentration-time curve from time 0 to the last experimental point measured (Clast); HL: plasma half-life of the terminal phase calculated by: $\mathrm{HL}=0.693 /$ ke. Table S3. 4-HPR and metabolites (oxo-4-HPR, MPR and DH-4-HPR) evaluated after oral Bio-nFeR single (day 1) or repeated daily treatment at dose of $150 \mathrm{mg} / \mathrm{kg}$. Cmax: maximum plasma concentration achieved after drug administration; Tmax: time until Cmax is reached; AUC last: experimental area under the concentration-time curve from time 0 to the last experimental point measured (Clast); HL: plasma halflife of the terminal phase calculated by: $\mathrm{HL}=0.693 / \mathrm{ke}$. Table S4. 4-HPR and metabolites concentrations measured after $24 \mathrm{~h}$ in feces of mice after acute treatment at different doses of Bio-nFeR (10, 50, 100 and 200 $\mathrm{mg} / \mathrm{Kg}$ ) (A) and after chronical treatment at dose of $150 \mathrm{mg} / \mathrm{kg}(\mathrm{B})$, in comparison with the NCl-FeR formulation. Table S5. 4-HPR and metabolites (MPR, oxo-4-HPR and DH-4-HPR) concentrations in plasma and tumors (lung cancer, melanoma and colon cancer) as in Fig. 4 BC. (PDF 308 kb)

\begin{abstract}
Abbreviations
4-HPR: 4-hydroxy(phenyl)retinamide); Bio-nFeR: Bionanofenretinide; BWL: body weight loss; CRC: colorectal cancer; CSC: cancer stem cells; FeR: fenretinide; HED: human equivalent doses; LC: lung cancer; MEL: melanoma; NCI-

Fer: National Cancer Institute fenretinide; NSG: NOD/SCID non-obese diabetic/ severe combined immunodeficiency gamma chain deficient; TUNEL: Terminal deoxynucleotidyl transferase dUTP nick end labeling
\end{abstract}

\section{Acknowledgments}

We thank Paola Di Matteo and Stefano Guida for providing general technical support and Massimo Macchia for support with animal care.

\section{Authors' contributions}

IO (conception and preparation of Bio-nFeR formulation, conception of experiments, critical review of the maniscript), VS (managing part of in vitro and most of the in vivo xenografts experiments, contribution in drafting the manuscript and figure editing), GS (cell viability studies/confocal experiments, critically revising the manuscript and figure editing), MZ (supervision of pharmacokinetcs studies and critical revision of the manuscritp), LB, AP and LZ (lipid metabolism studies, HPLC), FF (part of cell viability studies), MF, CM and EB (pharmacokinetic studies, 4HPR quantification), ADV (support with animal procedures), MF (technical support with confocal studies), MLDA and MB (part of in vivo experiments), GM (revision of manuscript), RDM (revision of manuscript), AZ (conception of the study, revising manuscript) AE (conception and design of the experiments, in vivo xenograft studies, drafting the manuscript). All authors read and approved the final manuscript. 


\section{Funding}

This work was supported by grants from the Italian Association for Cancer Research (Molecular Clinical Oncology Extension Program \#9979).

\section{Availability of data and materials}

The datasets generated and/or analysed during the current study are available from the corresponding author on reasonable request.

\section{Ethics approval and consent to participate}

Animal experimentation was conducted in conformance with the following laws, regulations, and policies governing the care and use of laboratory animals: Italian Governing Law (D. L. 26/2014; Authorization n.19/2008-A issued March 6, 2008 and Authorization n. 292/2015-PR issued on April 23, 2015 by the Ministry of Health).

\section{Consent for publication}

Not applicable.

\section{Competing interests}

The authors declare that they have no competing interests.

\section{Author details}

${ }^{1}$ Department of Pharmacy and Biotechnology, University of Bologna, Bologna, Italy. ${ }^{2}$ Department of Oncology and Molecular Medicine, Istituto Superiore di Sanità, Rome, Italy. ${ }^{3}$ Istituto di Patologia Generale, Università Cattolica del Sacro Cuore, Rome, Italy. ${ }^{4}$ Department of Oncology, Istituto di Ricerche Farmacologiche Mario Negri IRCCS, Milan, Italy. ${ }^{5}$ Department of Experimental Medicine, TOR, University of Rome "Tor Vergata", Rome, Italy. ${ }^{6}$ National Research Council of Italy (CNR), Institute of Translational Pharmacology IFT, Rome, Italy. ${ }^{7}$ Department of Ecological and Biological Sciences, University of Tuscia, Viterbo, Italy. ${ }^{8}$ Service for Biotechnology and Animal Welfare, Istituto Superiore di Sanità, Rome, Italy. ${ }^{9}$ National AIDS Center, Istituto Superiore di Sanità, Rome, Italy. ${ }^{10}$ Fondazione Policlinico Universitario "A. Gemelli" - I.R.C.C.S, Rome, Italy.

Received: 2 April 2019 Accepted: 13 August 2019

Published online: 22 August 2019

\section{References}

1. Bassani B, Bartolini D, Pagani A, Principi E, Zollo M, Noonan DM, et al. Fenretinide (4-HPR) targets Caspase-9, ERK 1/2 and the Wnt3a/beta-catenin pathway in Medulloblastoma cells and Medulloblastoma cell spheroids. PLoS One. 2016;11(7):e0154111.

2. Chen NE, Maldonado NV, Khankaldyyan V, Shimada H, Song MM, Maurer BJ et al. Reactive oxygen species mediates the synergistic activity of Fenretinide combined with the microtubule inhibitor ABT-751 against multidrug-resistant recurrent neuroblastoma xenografts. Mol Cancer Ther. 2016:15(11):2653-64

3. Children's Oncology G, Villablanca JG, Krailo MD, Ames MM, Reid JM, Reaman $\mathrm{GH}$, et al. Phase I trial of oral fenretinide in children with high-risk solid tumors: a report from the Children's Oncology group (CCG 09709). Journal of clinical oncology : official journal of the American Society of Clinical Oncology. 2006;24(21):3423-30.

4. Cooper JP, Hwang K, Singh H, Wang D, Reynolds CP, Curley RW Jr, et al. Fenretinide metabolism in humans and mice: utilizing pharmacological modulation of its metabolic pathway to increase systemic exposure. $\mathrm{Br}$ J Pharmacol. 2011;163(6):1263-75.

5. Date AA, Hanes J, Ensign LM. Nanoparticles for oral delivery: design, evaluation and state-of-the-art. J Control Release. 2016;240:504-26.

6. De Angelis, M., Francescangeli, F., La Torre, F., and Zeuner A. (2019). Stem cell plasticity and dormancy in the development of Cancer therapy resistance. [review]. Frontiers in Oncology.

7. Di Martile M, Desideri M, De Luca T, Gabellini C, Buglioni S, Eramo A, et al. Histone acetyltransferase inhibitor CPTH6 preferentially targets lung cancer stem-like cells. Oncotarget. 2016;7(10):11332-48.

8. Durante S, Orienti I, Teti G, Salvatore V, Focaroli S, Tesei A, et al. Anti-tumor activity of fenretinide complexed with human serum albumin in lung cancer xenograft mouse model. Oncotarget. 2014;5(13):4811-20.

9. Eramo A, Lotti F, Sette G, Pilozzi E, Biffoni M, Di Virgilio A, et al. Identification and expansion of the tumorigenic lung cancer stem cell population. Cell Death Differ. 2008;15(3):504-14.
10. Fettig LM, McGinn O, Finlay-Schultz J, LaBarbera DV, Nordeen SK, Sartorius CA. Cross talk between progesterone receptors and retinoic acid receptors in regulation of cytokeratin 5-positive breast cancer cells. Oncogene. 2017.

11. Field LD, Nag OK, Sangtani A, Burns KE, Delehanty JB. The role of nanoparticles in the improvement of systemic anticancer drug delivery. Ther Deliv. 2018;9(7):527-45.

12. Flemming A. Cancer stem cells: targeting the root of cancer relapse. Nat Rev Drug Discov. 2015;14(3):165.

13. Formelli F, Cavadini E, Luksch R, Garaventa A, Villani MG, Appierto V, et al. Pharmacokinetics of oral fenretinide in neuroblastoma patients: indications for optimal dose and dosing schedule also with respect to the active metabolite 4-oxo-fenretinide. Cancer Chemother Pharmacol. 2008;62(4):655-65

14. Francescangeli F, Patrizii M, Signore M, Federici G, Di Franco S, Pagliuca A, et al. Proliferation state and polo-like kinase1 dependence of tumorigenic colon cancer cells. Stem Cells. 2012;30(9):1819-30.

15. Garaventa A, Luksch R, Lo Piccolo MS, Cavadini E, Montaldo PG, Pizzitola $M R$, et al. Phase I trial and pharmacokinetics of fenretinide in children with neuroblastoma. Clinical cancer research : an official journal of the American Association for Cancer Research. 2003;9(6):2032-9.

16. Holliday MW Jr, Cox SB, Kang MH, Maurer BJ. C22:0- and C24:0dihydroceramides confer mixed cytotoxicity in T-cell acute lymphoblastic leukemia cell lines. PLoS One. 2013;8(9):e74768.

17. Hsu CY, Wang PW, Alalaiwe A, Lin ZC, Fang JY. Use of lipid Nanocarriers to improve Oral delivery of vitamins. Nutrients. 2019;11(1).

18. Iyer AK, Singh A, Ganta S, Amiji MM. Role of integrated cancer nanomedicine in overcoming drug resistance. Adv Drug Deliv Rev. 2013; 65(13-14):1784-802.

19. Jantratid E, Janssen N, Reppas C, Dressman JB. Dissolution media simulating conditions in the proximal human gastrointestinal tract: an update. Pharm Res. 2008;25(7):1663-76.

20. Kummar S, Gutierrez ME, Maurer BJ, Reynolds CP, Kang M, Singh H, et al. Phase I trial of fenretinide lym-x-sorb oral powder in adults with solid tumors and lymphomas. Anticancer Res. 2011:31(3):961-6.

21. Leon G, MacDonagh L, Finn SP, Cuffe S, Barr MP. Cancer stem cells in drug resistant lung cancer: targeting cell surface markers and signaling pathways. Pharmacol Ther. 2016;158:71-90.

22. Li Y, Rogoff HA, Keates S, Gao Y, Murikipudi S, Mikule K, et al. Suppression of cancer relapse and metastasis by inhibiting cancer stemness. Proc Natl Acad Sci U S A. 2015;112(6):1839-44.

23. Liu L, Li X, Chen L, Zhang X. Nanoscale functional biomaterials for Cancer Theranostics. Curr Med Chem. 2018;25(25):2987-3000.

24. Long P, Chen J, Wang D, Hu Z, Gao X, Li Z, et al. Influence of counterions on micellization of tetramethylammonium perfluorononanoic carboxylate in 1-butyl-3-methylimidazolium ionic liquid. J Phys Chem B. 2012;116(26): 7669-75.

25. Maeda H, Bharate GY, Daruwalla J. Polymeric drugs for efficient tumortargeted drug delivery based on EPR-effect. Eur J Pharm Biopharm. 2009; 71(3):409-19.

26. Maurer BJ, Kalous O, Yesair DW, Wu X, Janeba J, Maldonado V, et al. Improved oral delivery of $\mathrm{N}$-(4-hydroxyphenyl)retinamide with a novel LYM$X$-SORB organized lipid complex. Clinical cancer research : an official journal of the American Association for Cancer Research. 2007;13(10):3079-86.

27. Maurer BJ, Kang MH, Villablanca JG, Janeba J, Groshen S, Matthay KK, et al. Phase I trial of fenretinide delivered orally in a novel organized lipid complex in patients with relapsed/refractory neuroblastoma: a report from the new approaches to neuroblastoma therapy (NANT) consortium. Pediatr Blood Cancer. 2013;60(11):1801-8.

28. Maurer BJ, Melton L, Billups C, Cabot MC, Reynolds CP. Synergistic cytotoxicity in solid tumor cell lines between $\mathrm{N}$-(4hydroxyphenyl)retinamide and modulators of ceramide metabolism. J Natl Cancer Inst. 2000;92(23):1897-909.

29. Maurer BJ, Metelitsa LS, Seeger RC, Cabot MC, Reynolds CP. Increase of ceramide and induction of mixed apoptosis/necrosis by N-(4hydroxyphenyl)- retinamide in neuroblastoma cell lines. J Natl Cancer Inst. 1999;91(13):1138-46.

30. Meacham CE, Morrison SJ. Tumour heterogeneity and cancer cell plasticity. Nature. 2013;501(7467):328-37.

31. Mennucci, M. D. (1997). Continuum solvation models: a new approach to the problem of solute's charge distribution and cavity boundaries. [research article]. The Journal of Chemical Physics, 106, 5151. 
32. Mohrbacher AM, Yang AS, Groshen S, Kummar S, Gutierrez ME, Kang MH, et al. Phase I study of Fenretinide delivered intravenously in patients with relapsed or refractory hematologic malignancies: a California Cancer consortium trial. Clin Cancer Res. 2017:23(16):4550-5.

33. Mukherjee N, Reuland SN, Lu Y, Luo Y, Lambert K, Fujita M, et al. Combining a BCL2 inhibitor with the retinoid derivative fenretinide targets melanoma cells including melanoma initiating cells. J Invest Dermatol. 2015;135(3):842-50

34. Oberoi HS, Nukolova NV, Kabanov AV, Bronich TK. Nanocarriers for delivery of platinum anticancer drugs. Adv Drug Deliv Rev. 2013;65(13-14):1667-85.

35. Oridate N, Suzuki S, Higuchi M, Mitchell MF, Hong WK, Lotan R. Involvement of reactive oxygen species in $\mathrm{N}$-(4-hydroxyphenyl)retinamide-induced apoptosis in cervical carcinoma cells. J Natl Cancer Inst. 1997;89(16):1191-8.

36. Orienti I, Francescangeli F, De Angelis ML, Fecchi K, Bongiorno-Borbone L, Signore $\mathrm{M}$, et al. A new bioavailable fenretinide formulation with antiproliferative, antimetabolic, and cytotoxic effects on solid tumors. Cell Death Dis. 2019;10(7):529.

37. Pignatta S, Orienti I, Falconi M, Teti G, Arienti C, Medri L, et al. Albumin nanocapsules containing fenretinide: pre-clinical evaluation of cytotoxic activity in experimental models of human non-small cell lung cancer. Nanomedicine. 2015;11(2):263-73.

38. Rahmaniyan M, Curley RW Jr, Obeid LM, Hannun YA, Kraveka JM. Identification of dihydroceramide desaturase as a direct in vitro target for fenretinide. J Biol Chem. 2011;286(28):24754-64.

39. Reagan-Shaw S, Nihal M, Ahmad N. Dose translation from animal to human studies revisited. FASEB journal : official publication of the Federation of American Societies for Experimental Biology. 2008;22(3):659-61.

40. Roesch, A., Paschen, A., Landsberg, J., Helfrich, I., Becker, J. C., \& Schadendorf, D. (2016). Phenotypic tumour cell plasticity as a resistance mechanism and therapeutic target in melanoma. Eur J Cancer (Oxford, England : 1990), 59, 109-112.

41. Santini R, Pietrobono S, Pandolfi S, Montagnani V, D'Amico M, Penachioni $J Y$, et al. SOX2 regulates self-renewal and tumorigenicity of human melanoma-initiating cells. Oncogene. 2014;33(38):4697-708.

42. Sette G, Fecchi K, Salvati V, Lotti F, Pilozzi E, Duranti E, et al. Mek inhibition results in marked antitumor activity against metastatic melanoma patientderived melanospheres and in melanosphere-generated xenografts. J Exp Clin Cancer Res. 2013;32:91.

43. Sette G, Salvati V, Memeo L, Fecchi K, Colarossi C, Di Matteo P, et al. EGFR inhibition abrogates leiomyosarcoma cell chemoresistance through inactivation of survival pathways and impairment of CSC potential. PLoS One. 2012;7(10):e46891.

44. Sette G, Salvati V, Mottolese M, Visca P, Gallo E, Fecchi K, et al. Tyr1068phosphorylated epidermal growth factor receptor (EGFR) predicts cancer stem cell targeting by erlotinib in preclinical models of wild-type EGFR lung cancer. Cell Death Dis. 2015;6:e1850.

45. Shen S, Xia JX, Wang J. Nanomedicine-mediated cancer stem cell therapy. Biomaterials. 2016;74:1-18.

46. Siddique HR, Saleem M. Role of BMI1, a stem cell factor, in cancer recurrence and chemoresistance: preclinical and clinical evidences. Stem Cells. 2012;30(3):372-8

47. Todaro M, Gaggianesi M, Catalano V, Benfante A, lovino F, Biffoni M, et al. CD44v6 is a marker of constitutive and reprogrammed cancer stem cells driving colon cancer metastasis. Cell Stem Cell. 2014;14(3):342-56.

48. Ulukaya E, Kurt A, Wood EJ. 4-(N-hydroxyphenyl)retinamide can selectively induce apoptosis in human epidermoid carcinoma cells but not in normal dermal fibroblasts. Cancer Investig. 2001;19(2):145-54.

49. Veronesi U, De Palo G, Marubini E, Costa A, Formelli F, Mariani L, et al. Randomized trial of fenretinide to prevent second breast malignancy in women with early breast cancer. J Natl Cancer Inst. 1999;91(21):1847-56.

50. Vulcano F, Milazzo L, Ciccarelli C, Eramo A, Sette G, Mauro A, et al. Wharton's jelly mesenchymal stromal cells have contrasting effects on proliferation and phenotype of cancer stem cells from different subtypes of lung cancer. Exp Cell Res. 2016;345(2):190-8.

51. Wang H, Maurer BJ, Liu Y-Y, Wang E, Allegood JC, Kelly S, et al. N-(4Hydroxyphenyl)retinamide increases dihydroceramide and synergizes with dimethylsphingosine to enhance cancer cell killing. Mol Cancer Ther. 2008; 7(9):2967-76.

52. Xie H, Zhu F, Huang Z, Lee MH, Kim DJ, Li X, et al. Identification of mammalian target of rapamycin as a direct target of fenretinide both in vitro and in vivo. Carcinogenesis. 2012;33(9):1814-21.
53. Yan W, Du J, Du Y, Pu H, Liu S, He J, et al. Fenretinide targets the side population in myeloma cell line $\mathrm{NCl}-\mathrm{H} 929$ and potentiates the efficacy of antimyeloma with bortezomib and dexamethasone regimen. Leuk Res. 2016:51:32-40.

\section{Publisher's Note}

Springer Nature remains neutral with regard to jurisdictional claims in published maps and institutional affiliations.

\section{Ready to submit your research? Choose BMC and benefit from:}

- fast, convenient online submission

- thorough peer review by experienced researchers in your field

- rapid publication on acceptance

- support for research data, including large and complex data types

- gold Open Access which fosters wider collaboration and increased citations

- maximum visibility for your research: over $100 \mathrm{M}$ website views per year

At BMC, research is always in progress.

Learn more biomedcentral.com/submissions 